\title{
Some Physical Applications of Random Hierarchical Matrices
}

\author{
V. A. Avetisov ${ }^{a}$, A. Kh. Bikulov ${ }^{a}$, O. A. Vasilyev ${ }^{b, c}$, S. K. Nechaev ${ }^{d, e}$, and A. V. Chertovich $f$ \\ ${ }^{a}$ Semenov Institute of Chemical Physics, Russian Academy of Sciences, Moscow, 119991 Russia \\ ${ }^{b}$ Max-Planck-Institut für Metallforschung, D-70569, Stuttgart, Germany \\ ${ }^{c}$ Institut für Theoretische und Angewandte Physik, Universität Stuttgart, D-70569, Stuttgart, Germany \\ ${ }^{d}$ Lebedev Physical Institute, Russian Academy of Sciences, Moscow, 119991 Russia \\ ${ }^{e}$ Université Paris Sud, 91405, Orsay Cedex, France \\ ${ }^{f}$ Moscow State University, Moscow, 119992 Russia \\ e-mail: sergei.nechaev@gmail.com \\ Received March 20, 2009
}

\begin{abstract}
The investigation of spectral properties of random block-hierarchical matrices as applied to dynamic and structural characteristics of complex hierarchical systems with disorder is proposed for the first time. Peculiarities of dynamics on random ultrametric energy landscapes are discussed and the statistical properties of scale-free and polyscale (depending on the topological characteristics under investigation) random hierarchical networks (graphs) obtained by multiple mapping are considered.
\end{abstract}

PACS numbers: 05.40.a, 87.15.hg

DOI: $10.1134 / \mathrm{S} 1063776109090155$

\section{INTRODUCTION}

The heightened interest in statistical properties of ensembles of random matrices that arose in the 1950s was primarily due to a number of problems in nuclear physics. In particular, it was found that random matrices may serve as a simple model of neutron resonances of heavy nuclei, which correctly explains many of the observed statistical regularities in this field (the description of strongly excited states in terms of oneparticle models is an unsolvable problem). Soon it was found that the application of random matrices is not limited to only nuclear physics problems. Deep penetration of random matrices in the physics of condensed media has led to a breakthrough in understanding of the behavior of conductivity in disordered mesoscopic systems. The applications of the theory of random matrices were subsequently extended to statistical physics. Among the large number of publications in this field, we must specially mention recent works $[1,2]$ in which the statistics of limiting states of ensembles of random matrices and their physical applications are considered.

It should be recalled that a standard problem in the theory of random matrices is the calculation of the density of distribution of eigenvalues of random matrices and the distribution of intervals between eigenvalues under the assumption that all matrix elements are independent random quantities assuming values in a certain preset ensemble (see, for example, [3]). In such a formulation, the theory of random matrices is used for describing a wide range of physical phenomena; however, the theory in this form does not cover an important class of complex systems that can be described using the concept of hierarchical (ultrametric) organization $[4,5]$ of phase, dynamic, or structural states. Most often, the hierarchy of states emerges in many-particle systems of various origins with a large number of "frozen" constraints with different scales, which generate multidimensional hypersurfaces of potential energy (or free energy) with an astronomically large number of local minima.

Typical examples of such systems (which are often referred to as complex systems) are glasses and globular proteins. The hierarchical (ultrametric) concept as applied to such systems presumes that local minima of the energy landscape are clustered into hierarchically embedded basins of minima. Namely, each large basin consists of smaller basins each of which in turn contains embedded still smaller basins, and so on. Local minima basins are separated from one another by hierarchically ordered barriers (the smaller the basins, the "lower" the barriers separating them). Finally, it is assumed (which is a distinctive feature of the ultrametric concept) that the time of attainment quasi-equilibrium in any basin is much shorter than the time of escape from the given basin. In other words, the time of passage from one local minimum to another is controlled by the maximal barrier encountered on this path; i.e., the passage time obeys the "strong triangle inequality," viz., the inequality imposed on distances in a hierarchical system (such distances are referred to as ultrametric). Thus, the ultrametric concept is essentially the approximation of a multidimensional energy landscape by a hierarchical (ultrametric) land- 
scape and, accordingly, the approximation of stochastic dynamics of a complex system by a random process on an ultrametric space.

An ultrametric space of states is usually represented graphically as a branched $p$-adic Cayley tree with a branching index of $p+1$. The states themselves correspond to only the boundary of the "crown" of the tree, while the remaining part of the $p$-adic tree is a graph of ultrametric distances between the states and relations between embeddings of basins of states. Geometrical properties of an ultrametric space dramatically differ from the properties of the Euclidean space. Consequently, analysis of ultrametric models requires an adequate mathematical apparatus. Mathematical $p$ adic analysis offers rich opportunities in this respect [6]. However, it cannot be stated for sure that ultrametric models are basically incompatible with the models using conventional (say, Euclidean) metric properties. The relation between ultrametric and Euclidean models for some complex systems has recently become an object of discussion in the physics of disordered systems. In particular, the possibility of isometric embedding of a homogeneous Cayley tree into a three-dimensional Euclidean space was considered in [7]. On the other hand, it was shown in [8] that a random Gaussian energy landscape reproducing significant features of the dynamic behavior of the system on an ultrametric energy scale can be constructed in a Euclidean finite-dimensional space. Finally, a deeprooted relation between some ultrametric models of complex systems and tree models of disordered systems with a standard description free of the concept of ultrametricity will also be demonstrated in Section 2 of this article.

Apart from the theory of spin glasses, the ultrametric concept was implemented in several formal models of the so-called basin-to-basin kinetics developed for fluctuation and dynamic behavior of macromolecular systems [9-13]. These models reflect various aspects of an ultrametric random walk, viz., a uniform Markovian random process associated with jumplike transitions between local minima of the energy landscape on a regular basin hierarchy. Transition matrix $\bar{T}$ of an ultrametric random walk is symmetric and has a translation-invariant (along the principal diagonal) blockhierarchical structure similar to the Parisi matrix (see, for example, [4]). Eigenvalues $\left\{\bar{\lambda}_{\gamma}\right\}$ of the translationinvariant block-hierarchical kinetic matrix $\bar{T}$ are well known $[6,9,13]$,

$$
\bar{\lambda}_{\gamma}=-p^{\gamma} \bar{T}^{(\gamma)}-\left(1-p^{-1}\right) \sum_{\gamma^{\prime}=\gamma+1}^{\Gamma} p^{\gamma^{\prime}} \bar{T}^{\left(\gamma^{\prime}\right)},
$$

where $\bar{\lambda}_{0}=0$ by definition and $\bar{T}^{(\gamma)}$ is the matrix element corresponding to transitions between $\gamma$-level basins; the summation is carried out from level $\gamma+1$ to the maximal level $\gamma=\Gamma$. In particular, if the barrier height increases with $\gamma$ linearly, we have $\bar{T}^{(\gamma)}=$ $p^{-(\alpha+1) \gamma}, \alpha \geq 0$. This case is physically meaningful precisely for protein molecules $[13,14]$. The meaning of eigenvalues of the block-hierarchical kinetic matrix is clear: $\bar{\lambda}_{\gamma}$ is the probability of escape from a basin of level $\gamma$ via any of the above-lying levels. It should be noted that all energy basins for a regular basin hierarchy, which are associated with the same level $\gamma$, are identical from the energy point of view; for this reason, matrix elements $\bar{T}^{(\gamma)}$ and eigenvalues $\left\{\bar{\lambda}_{\gamma}\right\}$ of the translation-invariant block-hierarchical kinetic matrix depend only on hierarchical level $\gamma$.

In spite of the fact that translation-invariant kinetic matrices $\bar{T}$ have been successfully used recently to explain the experimentally observed fluctuationdynamic mobility of complex macromolecular objects like protein molecules [13, 14], a regular basin hierarchy appears as a strongly simplifying assumption that considerably limits the possibility of describing an actual physical system. The random nature of the basin hierarchy may change the behavior of the system so strongly that the approximation of the energy landscape by a regular hierarchy may prove not quite justified. To remove the degeneracy in energy inherent in a regular hierarchical landscape without distorting the basin structure of the landscape, it is natural to assume that the heights of the energy barriers belonging to the same hierarchical level are random and distributed in the vicinity of certain mean values controlled by a regular hierarchy.

Another argument in favor of randomization of the hierarchical landscape is associated with the fact that, in real experiments with micro- and mesoscopic scale objects, we are dealing as a rule not with a single object but with a macroscopic sample, viz., a statistical ensemble of objects with random variations of their individual landscapes. In this connection, manifestations of the hierarchical (ultrametric) organization of energy landscapes in the properties of ensemble-averaged become important. Analysis of this problem primarily involves the determination of the characteristics of an averaged spectrum of eigenvalues for the ensemble of random block-hierarchical kinetic matrices and the averaged relaxation function. This problem is considered in Section 2 of this article. To make the description all-sufficient, the main idea of calculating the eigenvalue spectrum for translation-invariant block-hierarchical kinetic matrices is given in the Appendix. In Section 2, we will also consider a number of interesting (in our opinion) questions that establish an unexpected relation between relaxation kinetics on random ultrametric landscapes and the critical phenomena on phase spaces of a tree structure [15].

Apart from the dynamic contents of the ultrametric paradigm, the determination of the hierarchical struc- 

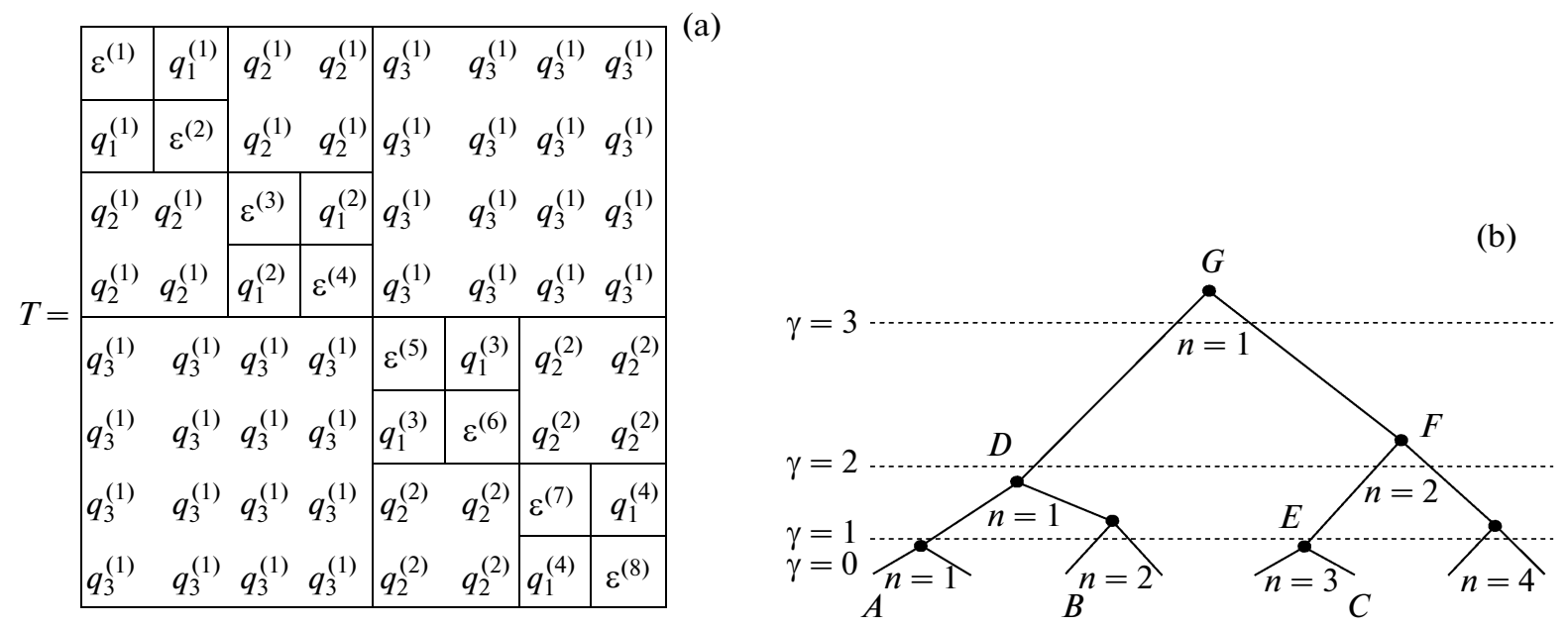

Fig. 1. (a) Block-hierarchical kinetic matrix $T$ of the Parisi type $(p=2)$; (b) hierarchical (ultrametric) energy landscape corresponding to kinetic matrix $T$.

ture of an organization of "random ultrametric phase spaces" in the observed statistical regularities is of considerable interest. A visual example of the hierarchical structural organization is the so-called crumpled globule discussed for the first time in [16]. The thermodynamically equilibrium spatial configuration of such a globule resembles the Peano curve [17] embedded into a 3D space. Spatial packing of a crumpled globule can be represented schematically by a single folded motive reproduced on a growing scale. The hierarchical packing naturally leads to a block-hierarchical network of contacts between the links of a chain described by a block-hierarchical matrix of contacts. Naturally, the presence of inhomogeneities in the hierarchy of crimps introduces randomicity in the blockhierarchical network of contacts, which requires the determination of statistical characteristics of an ensemble of random block-hierarchical matrices of contacts.

Analysis of spectral density $\rho(\lambda)$ for some ensembles of block-hierarchical matrices of contacts shows [18] that $\rho(\lambda)$ has a power (and not exponential) "tail" for $|\lambda| \longrightarrow \infty$. This indicates the polyscale nature of statistical characteristics of the network and suggests that networks (and the corresponding structures) with block-hierarchical matrices of contacts may belong the class of scale-free networks that are also referred to as scale-invariant, scale-self-similar, etc., networks.

The scale-free network structures are being considered in almost all fields of science from biopolymer dynamics and their spatial packing (folding) into strictly deterministic structures, cellular metabolism, and natural networks of various nature (including Internet, various ecological, social, and economic structures and objects) to bank networks and even Bose-Einstein condensation. Many items of this incomplete list are described in review [19]. For this reason, in Section 3, devoted to random networks, we concentrate our attention on several key features of block-hierarchical random networks; special attention will be paid to application of random block-hierarchical matrices of contacts (adjacency matrices). In Section 3, we also propose a new model of a hierarchical scale-free random network constructed using random block-hierarchical matrices of contacts. It should be noted that, although the structure of a block-hierarchical matrix of contacts is similar in many respects to the structure of the block-hierarchical kinetic matrix considered in Section 2, an important difference between these structures exists. In kinetic matrices, the diagonal elements satisfy by definition the condition of conservation of the total probability (at any instant, the system is for sure in one of the states), while the diagonal elements of block-hierarchical adjacency matrix are zero by definition. It will be demonstrated in Section 3 that this circumstance strongly affects the spectral properties of an ensemble of blockhierarchical matrices.

\section{RANDOM BLOCK-HIERARCHICAL KINETIC MATRICES: SPECTRAL PROPERTIES AND RELATION TO DIRECTED POLYMERS ON DISORDERED TREES}

Block-hierarchical kinetic matrices (Fig. 1a) are natural structures closely related to ultrametric random walk models. Let us consider an example of construction of such a block-hierarchical matrix. We take set of points $B_{p}(\Gamma)$ labeled by index $i=1, \ldots, N$, where $N=p^{\Gamma}$ ( $\Gamma$ is a fixed integer and $p$ is a prime number), and consider these points as a discrete lattice of sites over which a "particle" walks. To construct an ultrametric random walk on $B_{p}(\Gamma)$, we introduce a $p$-adic branching graph $G_{p}(\Gamma)$ with a fixed ramification index $p+1$ with lattice sites $B_{p}(\Gamma)$ as the finite vertices of the 
graph (Fig. 1b). Thus, graph $G_{p}(\Gamma)$ has $\Gamma$ hierarchical levels. We assume that probability $q(i, j)$ of transition between any two lattice sites $i$ and $j$ per unit time $(i, j \in$ $\left.B_{p}(\Gamma)\right)$ is controlled by the height of the smallest subtree $G_{p}\left(\gamma, n_{\gamma}\right) \subseteq G_{p}(\Gamma)$ including sites $i$ and $j$. In our notation, pair $\left(\gamma, n_{\gamma}\right)$ labels the points of subtree $G_{p}(\Gamma)$ : $1 \leq \gamma \leq \Gamma$ is the number of the hierarchical level at which the point of the subtree is located and $n_{\gamma}$ is the symbol assuming various values on the $p^{\Gamma-\gamma}$ vertices of level $\gamma$. Since the transition probabilities are defined by the vertices of the $p$-adic graph, the characteristic transition times satisfy the strong (ultrametric) triangle inequality and the $p$-adic graph can be associated with the ultrametric energy landscape. For example, the maximal activation barrier separating states $A$ and $B$ (see Fig. 1b) can be associated with the height at which vertex $D$ with indices $(\gamma=2, n=1)$ is located, and so on.

Thus, the transition matrix has the block-hierarchical structure which is directly related to the hierarchy of basins on the energy landscape. For brevity, we will refer to such a matrix as the Parisi kinetic matrix (PKM) and will denote its elements by $q_{\gamma}^{(n)}$, assuming that $n$ are the corresponding indices of matrix blocks (points of subtrees of a $p$-adic graphs) belonging to level $\gamma$. It should be noted that PKM is a symmetric matrix and its diagonal elements are defined so that the sum of elements in each column is zero.

It was noted above that each element $T_{i, j} \equiv q_{\gamma}^{(n)}$ of matrix $T$ (see Fig. 1a) can be related to the corresponding energy barrier $u_{\gamma}^{(n)}>0$ on the landscape separating a certain pair of basins of the corresponding scale: $u_{\gamma}^{(n)}=-\ln q_{\gamma}^{(n)}$ is the dimensionless barrier "height."

The eigenvalues of translation-noninvariant PKMs were obtained for the first time in [20] using elements of the $p$-adic analysis [6]. We will write below the expression for the PKM eigenvalues in terms of matrix elements $q_{\gamma}^{(n)}$. The eigenvectors of a translation-noninvariant PKM are described in the Appendix. The structure of $\lambda_{\gamma, n}$ has a clear geometrical interpretation. We define a pair of numbers $(\gamma, n)$, where $\gamma$ is a hierarchical level $(1 \leq \gamma \leq \Gamma=\max \gamma)$, while $n$ labels the vertices of the $p$-adic graph $\left(1 \leq n \leq p^{\Gamma-\gamma}\right)$ belonging to level $\gamma$ (see Fig. 1b). form

Eigenvalue $\lambda_{\gamma, n}$ of matrix $T$ can be written in the

$$
\lambda_{\gamma, n}=-p^{\gamma} q_{\gamma}^{(n)}-\left(1-p^{-1}\right) \underbrace{\sum_{\left(\gamma^{\prime}, n^{\prime}\right)=(\gamma+1, n)}^{(\Gamma, 1)} p^{\gamma^{\prime}} q_{\gamma^{\prime}}^{\left(n^{\prime}\right)}}_{\Sigma} .
$$

In spite of similarity with expression (1), summation in Eq. (2) requires explanation. This summation, which makes a contribution to eigenvalue $\lambda_{\gamma, n}$, is car- ried out along the (single) path on the tree connecting the vertex $(\Gamma, n)$ with the tree origin, while factor $p^{\gamma}$ in front of $q_{\gamma}^{(n)}$ is the number of states in the basin of level $\gamma$. Hence, it can easily be seen that eigenvalue $\lambda_{\gamma, n}$ is just the probability of escape (per unit time) from the subtree with point $(\gamma, n)$ through any of the abovelying points of the $p$-adic tree. By way of illustration, let us calculate eigenvalue $\lambda_{\gamma=1, n=3}$ for the matrix depicted in Fig. 1a. The first term in Eq. (2) is the weighted contribution from point $E(\gamma=1, n=3)$, and $\operatorname{sum} \Sigma$ in this case is the weighted sum of two contributions from points $F$ and $G$ (see Fig. 1b). It follows hence that

$$
\lambda_{1,3}=-2^{1} q_{1}^{(3)}-\left(1-2^{-1}\right)\left[2^{2} q_{2}^{(2)}+2^{3} q_{3}^{(1)}\right] .
$$

Let us now determine a random PKM. We assume that

$$
u_{\gamma}^{(n)}=\left\langle u_{\gamma}^{(n)}\right\rangle+\xi_{\gamma}^{(n)},
$$

where $\left\langle u_{\gamma}^{(n)}\right\rangle$ is the mean value of the barrier height at level $\gamma$ and $\xi_{\gamma}^{(n)}$ describes random deviations of $u_{\gamma}^{(n)}$ from its mean value. We will henceforth assume that $\left|\xi_{\gamma}^{(n)}\right| \ll 1$. This allows us to retain only the linear term in the power series for $q_{\gamma}^{(n)}$ in $\xi_{\gamma}^{(n)}$ :

$$
q_{\gamma}^{(n)} \approx \exp \left(-\left\langle u_{\gamma}^{(n)}\right\rangle\right)\left[1-\xi_{\gamma}^{(n)}+O\left(\left(\xi_{\gamma}^{(n)}\right)^{2}\right)\right] .
$$

It should be recalled that mean values $\left\langle u_{\gamma}^{(n)}\right\rangle$ fix the hierarchical structure of the energy landscape. We will assume for definiteness that the mean barrier height increases with $\gamma$ linearly; i.e., $\exp \left(-\left\langle u_{\gamma}^{(n)}\right\rangle\right)=\left\langle q_{\gamma}^{(n)}\right\rangle=$ $p^{-(\alpha+1) \gamma}(\alpha \geq 0)$, where $\alpha$ scales the linear (on average) increase in the barrier height over the landscape. It should be emphasized, however, that this does not restrict the extension of our analysis to other types of ultrametric landscapes discussed, for example, in [21].

Analysis of ultrametric random walk over randomized ultrametric landscape necessitates above all the calculation of the density of distribution of eigenvalues of a random PKM of the above type (see Fig. 1a). Apart from this problem, we will establish in this section the relation between randomized ultrametric landscapes and "directed polymers on random trees" [22]. Using this relation, we can observe some unexpected properties of the survival probability on random ultrametric landscapes. It should be recalled that survival probability $S(t)$ (also known as the relaxation function) is the probability of finding the system in the initial state at instant $t$.

\subsection{Spectral Density of a Random Parisi Kinetic Matrix}

Spectral density $\rho(\lambda)$ of an ensemble of random PKMs can be evaluated in the standard way: 


$$
\rho(\lambda)=\frac{1}{\mathcal{N}} \sum_{\{\gamma, n\}}\left\langle\delta\left(\lambda-\lambda_{\gamma, n}\right)\right\rangle,
$$

where $\mathcal{N}=p^{\Gamma}$ is the total number of eigenvalues of matrix $T$ and $\langle\ldots\rangle$ denotes averaging with distribution function $P\left(\xi_{\gamma}^{(n)}\right)$. Let $P\left(\xi_{\gamma}^{(n)}\right)$ be the distribution functions for the distribution of fluctuations $\xi_{\gamma}^{(n)}$ of barriers $u_{\gamma}^{(n)}$ belonging to level $\gamma$ (see expression (3)), and matrix elements $q_{\gamma}^{(n)}$ be connected with the energy barrier heights via relation (4). We assume that $P\left(\xi_{\gamma}^{(n)}\right)$ is a Gaussian distribution independent of $(\gamma, n)$ :

$$
P\left(\xi_{\gamma}^{(n)}\right)=\frac{1}{\sqrt{2 \pi \sigma^{2}}} \exp \left(-\frac{\left(\xi_{\gamma}^{(n)}\right)^{2}}{2 \sigma^{2}}\right) .
$$

Generally speaking, fluctuations $\xi_{\gamma}^{(n)}$ in relation (6) are unbounded and distribution $P\left(\xi_{\gamma}^{(n)}\right)$ is not always compatible with condition $\left|\xi_{\gamma}^{(n)}\right| \ll 1$. It should be recalled that the latter condition is important precisely for kinetic matrices since it must guarantee that matrix elements $q_{\gamma}^{(n)}$ in relation (4) are positive. Consequently, we will assume that $\left\langle\left(\xi_{\gamma}^{(n)}\right)^{2}\right\rangle=\sigma^{2} \ll 1$, calculate the spectral density, and then test our results for self-consistency.

Expressions (2), (5), and (6) readily give $\rho(\lambda)$. We begin with auxiliary computation of function $Q(\lambda, \gamma)=$ $\left\langle\delta\left(\lambda-\lambda_{\gamma, n=1}\right)\right\rangle$ for $n=1$ and for an arbitrary value of $\gamma(1 \leq \gamma \leq \Gamma)$, where averaging is carried out with distribution $P\left(\xi_{\gamma}^{(n=1)}\right)$ :

$$
\begin{gathered}
Q(\lambda, \gamma)=\int_{-\infty}^{\infty} \frac{d x}{2 \pi} e^{i \lambda x}\left\langle e^{-i \lambda_{\gamma, n} x}\right\rangle_{\left\{P\left(\xi_{\gamma}^{(n=1)}\right), \ldots, P\left(\xi_{\Gamma}^{(n=1)}\right)\right\}} \\
\approx \int_{-\infty}^{\infty} \frac{d x}{2 \pi} \exp \left\{i x\left[\lambda+v_{\gamma}(\Gamma)\right]-\frac{x^{2}}{2} \sigma^{2} u_{\gamma}(\Gamma)\right\},
\end{gathered}
$$

where

$$
\begin{aligned}
& v_{\gamma}(\Gamma)=p^{-\alpha \gamma}+\left(1-p^{-1}\right) \sum_{\gamma^{\prime}=\gamma+1}^{\Gamma} p^{-\alpha \gamma^{\prime}} \\
& u_{\gamma}(\Gamma)=p^{-2 \alpha \gamma}+\left(1-p^{-1}\right)^{2} \sum_{\gamma^{\prime}=\gamma+1}^{\Gamma} p^{-2 \alpha \gamma^{\prime}}
\end{aligned}
$$

The integral in expression (7) can be evaluated easily. Retaining only the principal (quadratic) terms in the expansion in $\sigma$, we obtain

$$
Q(\lambda, \gamma)=\frac{1}{\sqrt{2 \pi \sigma^{2} u_{\gamma}(\Gamma)}} \exp \left\{-\frac{\left[\lambda+v_{\gamma}(\Gamma)\right]^{2}}{\sigma^{2} u_{\gamma}(\Gamma)}\right\}
$$

Note that quantity $Q(\lambda, \gamma)$ is the same for any of $p^{\Gamma-\gamma}$ eigenvalues $\lambda_{\gamma, n}$ corresponding to $p^{\Gamma-\gamma}$ basins of level $\gamma$. Consequently, we can write spectral density $\rho(\lambda)$ in the form

$$
\rho(\lambda)=\frac{1}{p^{\Gamma}} \sum_{\gamma=1}^{\Gamma} g(\gamma) Q(\lambda, \gamma),
$$

where $g(\gamma)=p^{\Gamma-\gamma}$ is the degeneracy of quantity $Q(\lambda, \gamma)$.

To verify the correctness of approximation (4), we have determined numerically the spectral density $\rho(\lambda)$ for the Gaussian distribution $P\left(\xi_{\gamma}^{(n)}\right)$ using the exact relation $q_{\gamma}^{(n)}=p^{-(\alpha+1) \gamma} e^{-\xi_{\gamma}^{(n)}}$ and compared the result with the spectral density obtained from the linear approximation $q_{\gamma}^{(n)} \approx p^{-(\alpha+1) \gamma}\left(1-\xi_{\gamma}^{(n)}\right)$. Figure $2 \mathrm{a}$ shows the exact and approximate spectral density distributions obtained for random PKMs for $\Gamma=8$ and $\alpha=0.1$. Analogous results for $\alpha=0.5$ are depicted in Fig. 2b. It can be seen that, for $\sigma \lesssim 0.2$, linearized approximation (4) for a Gaussian energy barrier distribution is still meaningful: the approximate value of $\rho(\lambda)$ calculated using expressions (9) and (10) almost coincides with the actual distribution.

Finally, it should be noted that analysis of expression (10) for an arbitrary $\alpha>0$ in the limit $\Gamma \longrightarrow \infty$ allows us to determine the asymptotic behavior of the tail of spectral density $\rho(\lambda)$ for $\lambda \longrightarrow-\infty$ :

$$
\begin{gathered}
\left.\rho(\lambda)\right|_{\lambda \rightarrow-\infty} \\
\approx \begin{cases}|\lambda|^{-(\alpha-1) / \alpha} e^{-\lambda^{2}}, & \alpha \geq 1 \\
|\lambda|^{-(1-\alpha) / \alpha} e^{-\lambda^{2}}, & 0<\alpha<1 .\end{cases}
\end{gathered}
$$

Strong truncation of the spectrum on the left for small values of $\sigma$ (see Fig. 2) (i.e., for weak randomization of the ultrametric energy landscape) indicates that physically interesting manifestations of the landscape randomicity against the background of the clearly manifested hierarchical basin structure should be expected only for the part of the random PKM spectrum corresponding to long time intervals and to the domain of $\lambda \longrightarrow 0$. This question will be discussed in detail in the next section.

\subsection{Survival Probability on a Random Ultrametric Landscape and Directed Polymers on Random Trees}

Information on the behavior of the system over long time periods can be gained from survival probability $S(t)$, viz., the probability of funding the system in the initial state at instant $t$. The survival probability on regular ultrametric landscapes (described by regular $p$ - 

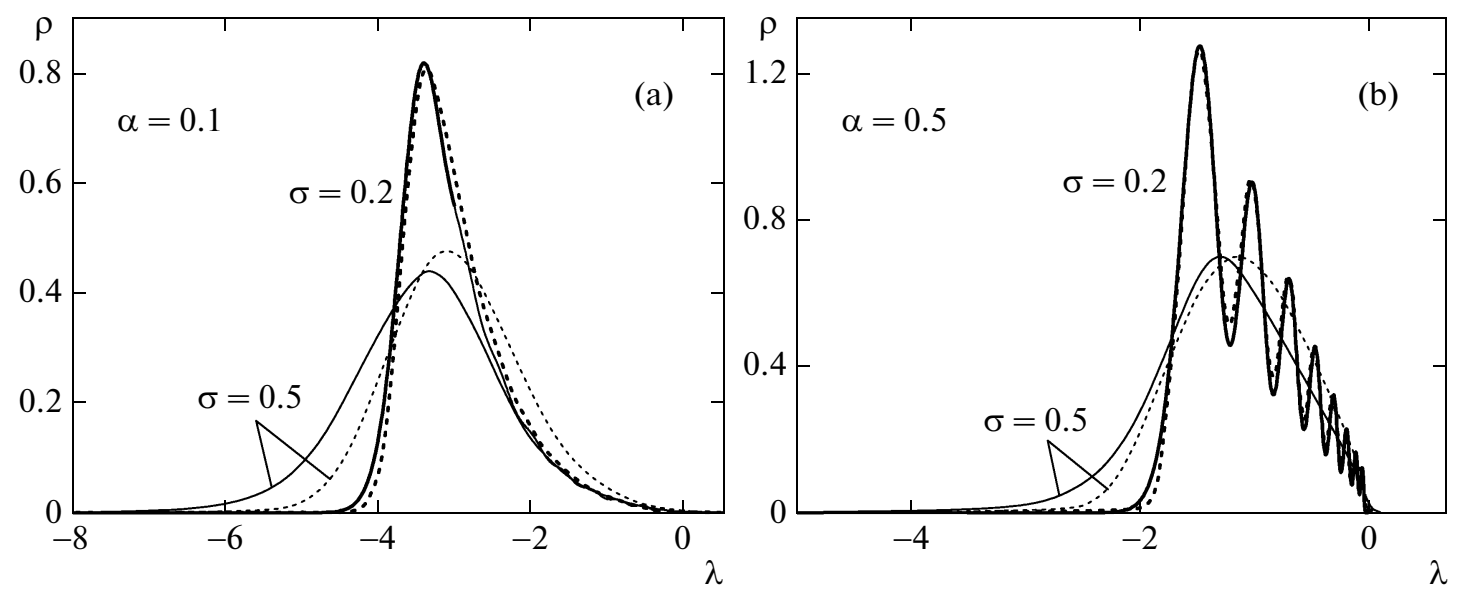

Fig. 2. Spectral density distribution $\rho(\lambda)$ of a PKM with Gaussian fluctuations of barrier heights for exact (solid curves) and linearized (dotted curves) matrix elements for $\Gamma=8, \alpha=0.1$ (a), 0.5 (b), and $\sigma=0.2$ and 0.5.

adic graphs) is known for various types of landscapes (see, for example, [21]):

$$
\bar{S}(t, \Gamma)=(p-1) \sum_{\gamma=1}^{\Gamma} p^{-\gamma} e^{\lambda_{\gamma} t}+p^{-\Gamma},
$$

where eigenvalues $\lambda_{\gamma}<0, \gamma=1, \ldots, \Gamma$. It should be noted that the initial distribution on a regular ultrametric landscape always evolves to a uniform distribution. As regards the survival probability, it depends on the landscape profile. For example, for linear landscapes (on which the height of activation barriers between basins increases linearly with $\gamma)$, the eigenvalues of the PKM are defined by expression (1), and $S(t)$ behaves over long time periods as $S(t) \propto t^{-1 / \alpha}[9,21]$.

For an ultrametric random walk with a random PKM, survival probability $S(t)$ depends on the specific implementation of the PKM. It was noted above that in this case the behavior of survival probability $\langle S(t, \Gamma)\rangle$ averaged over implementations of the PKM over long time intervals is interesting from the physical point of view:

$$
\begin{gathered}
\langle S(t, \Gamma)\rangle=\left\langle(p-1) \sum_{\{\gamma, n\}} p^{-\gamma} \exp \left(\lambda_{\gamma, n} t\right)\right\rangle \\
=(p-1) \sum_{\gamma=1}^{\Gamma} p^{-\gamma}\langle Z(t, \gamma, \Gamma)\rangle,
\end{gathered}
$$

where

$$
Z(t, \gamma, \Gamma)=\sum_{n=1}^{p^{\Gamma-\gamma}} \exp \left(\lambda_{\gamma, n} t\right)
$$

Summation in Eq. (13) is carried out over $p^{\Gamma-\gamma}$ points of subtrees of level $\gamma$, and $\lambda_{\gamma, n}$ is defined by expression (2). It should be recalled that each eigenvalue $\lambda_{\gamma, n}$ of a random PKM is the probability of escape (per unit time) from the basin corresponding to the subtree with point $(\gamma, n)$ (see Fig. 1b). Consequently, we can formally treat $Z(t, \gamma, \Gamma)$ as the statistical sum over all paths of departure from level $\gamma$ to upper-lying levels at instant $t$.

Direct calculation of $\langle S(t, \Gamma)\rangle$ is a complicated problem; however, important information on the behavior of $\langle S(t, \Gamma)\rangle$ can be obtained from analysis of the distribution function for $Z(t, \gamma, \Gamma)$. Further analysis of this problem will be based on the observation that $Z(t, \gamma, \Gamma)$ formally coincides with the statistical sum of a directed polymer on a disordered tree (DPDT), which was studied for the first time in [22].

Substituting expression (4) into (13) and retaining only linear terms in $\xi_{\gamma}^{(n)}\left(\left|\xi_{\gamma}^{(n)}\right| \ll 1\right)$, we obtain

$$
Z(t, \gamma, \Gamma)=\tilde{Z}(t, \gamma, \Gamma) \exp \left[-v_{\gamma}(\Gamma) t\right]
$$

where $v_{\gamma}(\Gamma)$ is defined by formula $(8)$ and

$$
\begin{aligned}
& \tilde{Z}(t, \gamma, \Gamma)=\sum_{\text {all paths }} \exp \left\{t p^{-\alpha \gamma} \xi_{\gamma}^{(n)}\right. \\
& \left.\left.+\left(1-p^{-1}\right) \sum_{\left(\gamma^{\prime}=\gamma+1, n\right)}^{(\Gamma, 1)} p^{-\alpha \gamma^{\prime}} \xi_{\gamma^{\prime}}^{\left(n^{\prime}\right)}\right)\right\} .
\end{aligned}
$$

Summation in each exponent on the right-hand side of this expression is carried out over all points lying on the path from vertex $(\gamma+1, n)$ to the three origin $(\Gamma, 1)$ (exactly as in formula (2)), while the first summation in Eq. (14) is performed over all $p^{\Gamma-\gamma}$ points of the $p$ adic tree belonging to level $\gamma$.

Function $\tilde{Z}(t, \gamma, \Gamma)$ satisfies the following stochastic recurrence equation (cf. the equation derived in [22]):

$$
\tilde{Z}(\Gamma)=\exp \left[t\left(1-p^{-1}\right) p^{-v \Gamma} \xi_{\Gamma}\right] \sum_{j=1}^{p} \tilde{Z}_{j}(\Gamma-1) .
$$


Following [22], we introduce the averaged characteristic (generating) function

$$
\begin{gathered}
G_{m}(x) \\
=\left\langle\exp \left\{-\tilde{Z}(m) \exp \left[t\left(1-p^{-1}\right) p^{-\alpha m} x\right]\right\}\right\rangle,
\end{gathered}
$$

where $\gamma \leq m \leq \Gamma$. Using the factorization of function $G_{m}(x)$ on the Cayley tree, we arrive at the following recursion for function $G_{m}(x)$ :

$$
G_{m-1}(x)=\int d \xi P(\xi)\left[G_{m}\left(p^{-\alpha}(x+\xi)\right)\right]^{p},
$$

where $P(\xi)$ is Gaussian distribution (6) for $\xi$. To attach expression (17) to the boundary condition, we set $\tilde{Z}(t, \gamma=\Gamma, \Gamma)=\exp \left(t p^{-\alpha \Gamma} \xi_{\Gamma}\right)$ in formula (14). Thus, we fix the boundary condition at the origin of the tree:

$$
\begin{aligned}
G_{\Gamma}(x)= & \left\langle\operatorname { e x p } \left\{-\exp \left[t\left(1-p^{-1}\right) p^{-\alpha \Gamma} x\right]\right.\right. \\
& \left.\left.\times \exp \left[t p^{-\alpha \Gamma} \xi_{\Gamma}\right]\right\}\right\rangle_{P\left(\xi_{\Gamma}\right)} .
\end{aligned}
$$

The linear approximation in expression (14) suggests that distribution $P(\xi)$ is narrow with $\sigma^{2} \ll 1$. In this case, boundary condition (18) has the form

$$
G_{\Gamma}(x)=\exp \left\{-\exp \left[t\left(1-p^{-1}\right) p^{-\alpha \Gamma} x\right]\right\} .
$$

Equations (17) and (19) define our problem. To compare further the DPDT model and relaxation on random ultrametric landscapes, it is more convenient to reverse the readout direction for tree levels by defining $n=\Gamma-m, 0 \leq n \leq \Gamma-\gamma$. The case of $\alpha>0$ corresponds to a "contracting" mapping and requires separate analysis; here, we consider the case of $\alpha=0$ (i.e., $\sigma_{\gamma}=p^{-\gamma}$ ), which is formally identical to the DPDT model [22]. According to [22], Eq. (17) with boundary conditions (19) in the continuous limit corresponds to the Kolmogorov-Petrovsky-Piscounov equation [23] and has a solution of the traveling wave type.

The main property of the DPDT model can be described as follows. For long paths on the Cayley tree, $G_{n}(x)$ is a "traveling wave," $G_{n}(x)=w(x-f n)$, where velocity $f$ of propagation is a function of reciprocal temperature $\beta$. In terms of our model, $\beta=t\left(1-p^{-1}\right)$ $($ for $\alpha=0$ ). In the DPDT model, velocity $f$ is an analog of the free energy of a directed polymer per unit length and has the following form in the limit of large lengths:

$$
f(\beta)=\frac{1}{\beta} \ln \left(p \int d \xi P(\xi) e^{\beta \xi}\right) .
$$

At high temperatures $T>T_{c}$ (i.e., at $t<t_{c}$ in terms of Eqs. (17) and (19)), velocity $f$ of a traveling wave (i.e., the free energy of the directed polymer) varies with temperature. However, at low temperatures $T<T_{c}$ (i.e., at $t>t_{c}$ in terms of our model), velocity $f$ is constant and equal to $f\left(T_{c}\right)\left(f\left(t_{c}\right)\right.$ in terms of our model). The value of $T_{c}$ is defined by the solution to the equation

$$
\left.\frac{d}{d t} f(T)\right|_{T=T_{c}}=0
$$

Since the temperature dependence of free energy changes as we pass through temperature $T=T_{c}$, we can naturally assume that the "ergodicity breakdown" phase transition [24] takes place at point $T_{c}$; i.e., the tree-like phase space of the directed polymer splits into separate domains. Indeed, it was noted in [22] that the overlap of two states of a directed polymer (paths on a tree) depends on temperature. For $T>T_{c}$, the overlap is zero for sure; however, for $T<T_{c}$, it is zero with a probability $\pi(t) \propto T$ and unity with a probability of $1-\pi(t)$. A nonzero overlap probability indicates that, below the critical temperature $T_{c}$, the phase space of a directed polymer becomes inhomogeneous. In our case, the solution to Eq. (17) behaves analogously over long time intervals on account of the fact that time $t$ plays the role of reciprocal temperature $\beta$ of the DPDT model. Proceeding from the formal analogy, we could assume that even a weak randomization of the ultrametric landscape $\left(\left|\xi_{\gamma}^{(n)}\right| \ll 1\right)$ may significantly change the behavior of a walking particle over long time intervals $\left(t>t_{c}\right)$. It should be noted, however, that the case where $\alpha \longrightarrow 0$ corresponds to a vanishing barrier hierarchy (with preserved hierarchy of basins); consequently, "weak" fluctuations $\left|\xi_{\gamma}^{(n)}\right| \ll 1$ (as compared to vanishingly small mean values $\left\langle u_{\gamma}^{(n)}\right\rangle$ ) are in fact "strong" fluctuations. In other words, the case of $\alpha=0$ and $\left|\xi_{\gamma}^{(n)}\right| \ll 1$ corresponds to strong fluctuations of the barrier hierarchy.

In spite of the fact that our problem for $\alpha=0$ formally corresponds to the DPDT model completely, the interpretation of the critical phenomenon observed in [22] in terms of ultrametric random walk [15] deserves discussion. Indeed, our kinetic problem is defined for an ultrametric random walk (i.e., for the walk over the boundary of a $p$-adic tree), and all kinetic properties of the model (including the "traveling wave") are defined by the probabilities of transition over certain (ultrametric) distances "across the tree" along its boundary. Conversely, the DPDT model deals with a walk in the bulk of the tree, and a traveling wave propagates "along the tree" from its origin to the boundary.

Nevertheless, the trajectory overlap calculated in [22] for a directed polymer allows us to relate the behavior in the bulk of the tree and the behavior on its boundary. Since the overlap of any two trajectories on the tree is zero for $t<t_{c}$, the tree boundary (to which the trajectories come from the origin) remains kinetically uniform in the sense that the probability of departure from any basin averaged over all realizations of a random landscape depends only on the scale (entropy) of a basin. In other words, over not very long time periods, the observed kinetics is analogous to the kinetics on the regular basin hierarchy, and randomicity of the ultrametric landscape is not manifested in any way. Conversely, the overlap of trajectories over 
long time periods $t>t_{c}$ indicates statistical nonuniformity of the contributions from different realizations of a random ultrametric landscape to the behavior of relaxation mean values: the tree boundary remains homogeneous with a probability $\pi(t) \propto t^{-1}$, but becomes "lacunary" with a probability of $1-\pi(t)$; i.e., the boundary becomes such that the escape from some basins is (on average) kinetically hindered and a randomly walking particle may be trapped in these basins for an indefinitely long time. The probability of kinetic "freezing" in this case increases with time. Such relaxation properties on random landscapes with the basin structure may be easily observable, being manifested, for example, in the form of aging effects.

\section{RANDOM BLOCK-HIERARCHICAL MATRICES OF CONTACTS: SPECTRAL PROPERTIES AND RELATION WITH SCALE-FREE RANDOM NETWORKS}

It was noted in the Introduction that the determination of the structural organization of a complex system with statistical disorder is often based on the network paradigm, viz., on topological characteristics of the network of links obtained from analysis of interactions between elements of the system. Analytic approaches to the solution of such a problem are usually based on the relation between topological characteristics of the random network and the spectral properties of adjacency matrices (matrices of contacts) of the network. To analyze the spectral properties of adjacency matrices of completely random networks known as Erdös-Rényi random graphs (ER graphs) [25], an arsenal of methods both mathematically rigorous and using less stringent approaches borrowed from the theory of disordered systems exists (e.g., the replica technique or the Flori mean field method [26, 27]).

At the same time, vast data on statistical characteristics of real networks of various origins (from intermolecular networks of links in biopolymers to social financial networks and Internet) have been obtained in the last decade mainly owing to considerable advances in computer technology. It turned out that the topological properties of real networks and the spectral properties of their adjacency matrices do not match the characteristics of completely random ER graphs in most cases. It was found that a large number of real networks exhibit a power (and not exponential as in the case of ER graphs) behavior of the tails of the probability distributions of network characteristics; for this reason, such networks were singled out into a special class and are known as scale-free networks. In actual practice, the scale-free nature of a specific network is established in most cases from the vortex degree distribution (the number of the nearest neighbors for the vertices of the network under investigation). In the general formulation based on analysis of the properties of the density distribution for eigenvalues of the network adjacency matrix, we will identify the scale-free nature with the power behavior of precisely the spectral density. The topological characteristics of the network (e.g., vertex degree) have very broad distributions with a width exceeding by several orders of magnitude the widths corresponding to distributions for ER graphs. The relevant anomalously broad distributions will be referred to as polyscale distributions.

We can apparently state without exaggerating that the block-hierarchical order is typical rather than exotic of many complex systems combining polyscale behavior and randomicity. Examples of such systems can easily be found in various fields of mathematics, physics, and biology (from chaotic mapping in Hamiltonian systems $[28,29]$ to globular structures of polyelectrolytic networks [30] and hierarchical organization of biopolymers [31]). By way of such example, we will consider below the possibility of implementation of block-hierarchical matrices of contacts in globular structures of polymer molecules with topological interactions.

It is well known that the nonphantom nature of polymer chains leads to two types of interactions: bulk interactions vanishing in infinitely thin chains and topological interactions, which are preserved even for zero-thickness chains. At high temperatures, a polymer molecule has a strongly fluctuating ball structure without a well-defined thermodynamic state. However, at temperatures below a certain critical value $\Theta$, a macromolecule collapses into a weakly fluctuating drop-shaped globular structure. It was shown in classical works [32, 33] devoted to analysis of a ball-globule transformation free of topological interactions that the globular state at $T<\Theta$ can be described in the virial approximation taking into account two- and threeparticle interactions, $B=b(T-\Theta) / \Theta<0$ and $C=$ const $>0$ (see, for example, $[34,35])$. The approach developed in $[32,33]$ forms the basis of the contemporary statistical physics of the globular state, which is typical, for example, of most biopolymers.

In the globular phase formed by an unknotted macromolecule, topological limitations play the role of auxiliary repulsion. In a poor solvent (i.e., at $T<\Theta$ ), there exists a certain critical length $g^{*}$ depending on temperature and bulk interactions such that chains longer than $g^{*}$ collapse. For long polymer chains, we can define these $g^{*}$-link segments as new block monomers or the first-level folds (Fig. 3a). The chain segment containing several consecutive block folds may "collapse" in its own volume, forming a second-level fold; then second-level folds form a third-level fold, and so on. This process of hierarchical packing of the entire chain is completed when all $g^{*}$-linked segments of the chain are inside a single fold. Figure 3a shows three consecutive stages of formation of a folded globular structure [16]. Pay attention to the fact that the curve depicting the polymer chain packed in accordance with the above procedure resembles a self-similar Peano curve embedded into a 3D space. 
(a)

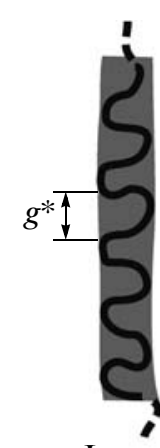

(a)
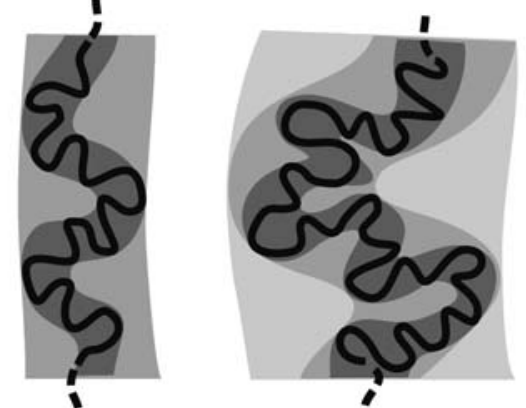

I

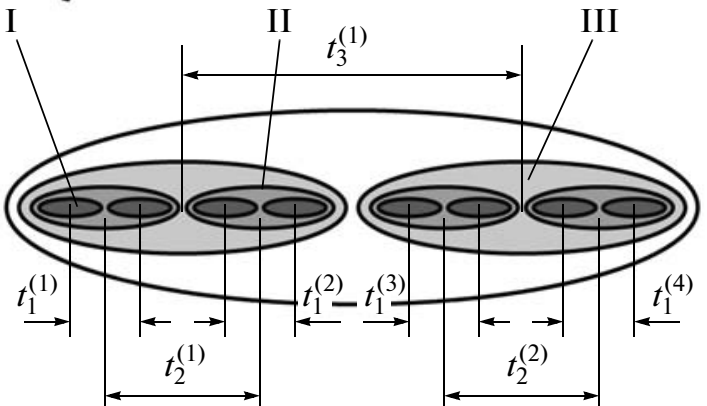

(b)

\begin{tabular}{|c|c|c|c|c|c|c|c|}
\hline 0 & $t_{1}^{(1)}$ & & & \multirow{4}{*}{$\begin{array}{l}t_{3}^{(1)} \\
t_{3}^{(1)} \\
t_{3}^{(1)} \\
t_{3}^{(1)}\end{array}$} & \multirow{3}{*}{$\begin{array}{l}t_{3}^{(1)} \\
t_{3}^{(1)} \\
t_{2}^{(1)}\end{array}$} & \multirow{2}{*}{$\begin{array}{l}t_{3}^{(1)} \\
t_{3}^{(1)}\end{array}$} & \multirow{2}{*}{$\begin{array}{l}t_{3}^{(1)} \\
t_{3}^{(1)}\end{array}$} \\
\hline$t_{1}^{(1)}$ & 0 & $t_{2}^{(1)}$ & $t_{2}^{(1)}$ & & & & \\
\hline$t_{2}^{(1)}$ & $t_{2}^{(1)}$ & 0 & $t_{1}^{(2)}$ & & & $t_{3}^{(1)}$ & $t_{3}^{(1)}$ \\
\hline$t_{2}^{(1)}$ & $t_{2}^{(1)}$ & $t_{1}^{(2)}$ & 0 & & $t_{3}^{(1)}$ & $t_{3}^{(1)}$ & $t_{3}^{(1)}$ \\
\hline$t_{3}^{(1)}$ & $t_{3}^{(1)}$ & $t_{3}^{(1)}$ & $t_{3}^{(1)}$ & 0 & $t_{1}^{(3)}$ & $t_{2}^{(2)}$ & $t_{2}^{(2)}$ \\
\hline$t_{3}^{(1)}$ & $t_{3}^{(1)}$ & $t_{3}^{(1)}$ & $t_{3}^{(1)}$ & $t_{1}^{(3)}$ & 0 & $t_{2}^{(2)}$ & $t_{2}^{(2)}$ \\
\hline$t_{3}^{(1)}$ & $t_{3}^{(1)}$ & $t_{3}^{(1)}$ & $t_{3}^{(1)}$ & $t_{2}^{(2)}$ & $t_{2}^{(2)}$ & 0 & $t_{1}^{(4)}$ \\
\hline$t_{3}^{(1)}$ & $t_{3}^{(1)}$ & $t_{3}^{(1)}$ & $t_{3}^{(1)}$ & $t_{2}^{(2)}$ & $t_{2}^{(2)}$ & $t_{1}^{(4)}$ & 0 \\
\hline
\end{tabular}

Fig. 3. (a) Three consecutive stages of formation of a block-hierarchical folded globule: folds of the first (I), second (II), and third (III) levels; (b) random block-hierarchical $p$-adic Parisi matrix $T$ with $p=2$.

A specific feature of the folded globule is that the block-hierarchical system of folds is not destroyed and is in thermodynamic equilibrium. The scale of the folds shown by shades of gray in Fig. 3a can be treated as the scale of truncation of interactions between $g^{*}$ linked block "monomers" of the chain. Interaction constants $t_{\gamma}^{(n)}$ correspond to the interaction between $g^{*}$-linked block monomers in a fold of hierarchical level $\gamma$. It can easily be seen that quantities $t_{\gamma}^{(n)}$ defined in this way form a block-hierarchical matrix $T$ depicted in Fig. $3 b$.

It was shown in [16] (and more rigorously in a later publication [36]) that the absence of knots in a closely packed folded polymer globule leads to peculiar fractal properties of the curve representing the polymer chain, which in turn strongly changes the thermodynamic properties of the globular state. The folded globule model was used for describing a self-similar hierarchical structure in biopolymers like DNA [37] and chromatin [38]; however, we can easily find other examples of biological systems in which the order has the form of a block-hierarchical structure of bonds of physical and chemical origin.

We will discuss below some topological properties of block-hierarchical random networks. On the basis of the results, we will propose a new method for growing polyscale networks that does not involve the control of the current state of the network.

\section{1. "Heavy Tails" in the Spectral Density of Gaussian Block-Hierarchical Adjacency Matrices}

Let us describe the general procedure of constructing a random hierarchical network. We set $N=p^{\Gamma}$ points which are potential vertices of our future network. The hierarchical network can be obtained by connecting vertices by links in a certain way. The information on the method of connection is contained in the $N \times N$ adjacency matrix $T$ that codes the presence and absence of links between the vertices. Namely, matrix element $T_{i, j}$ is unity if vertices $i$ and $j$ are directly connected by a link; otherwise, $T_{i, j}=0 ; T_{i, j}=$ $T_{j, i}$ and $T_{i, i}=0$. We construct the adjacency matrix in the form of a random block-hierarchical ( $p$-adic) matrix of the Parisi matrix type (PAM is the adjacency matrix of the Parisi matrix type). Such a matrix is shown in Fig. $3 \mathrm{~b}$ for $p=2$. Matrix elements $T_{i, j} \equiv t_{\gamma}^{(n)}$ are random quantities with the Bernoulli distribution:

$$
t_{\gamma}^{(n)}=\left\{\begin{array}{lll}
1 & \text { with probability } & q_{\gamma} \\
0 & \text { with probability } & 1-q_{\gamma},
\end{array}\right.
$$

where index $\gamma$ labels a hierarchical level as in Parisi kinetic matrices (PKM) considered above $(1 \leq \gamma \leq$ $\gamma_{\max } \equiv \Gamma$ ), while index $n$ labels the matrix blocks corresponding to hierarchical level $\gamma$. It should be noted that, in our construction, probability $q_{\gamma}$ of the link formation depends only on the scale of blocks (i.e., on the number of level $\gamma$ ) and is independent of $n$. The distribution of matrix elements $t_{\gamma}^{(n)}$ of $N \times N$ matrix $T$, where $N=p^{\Gamma}$, is completely controlled by the set of 

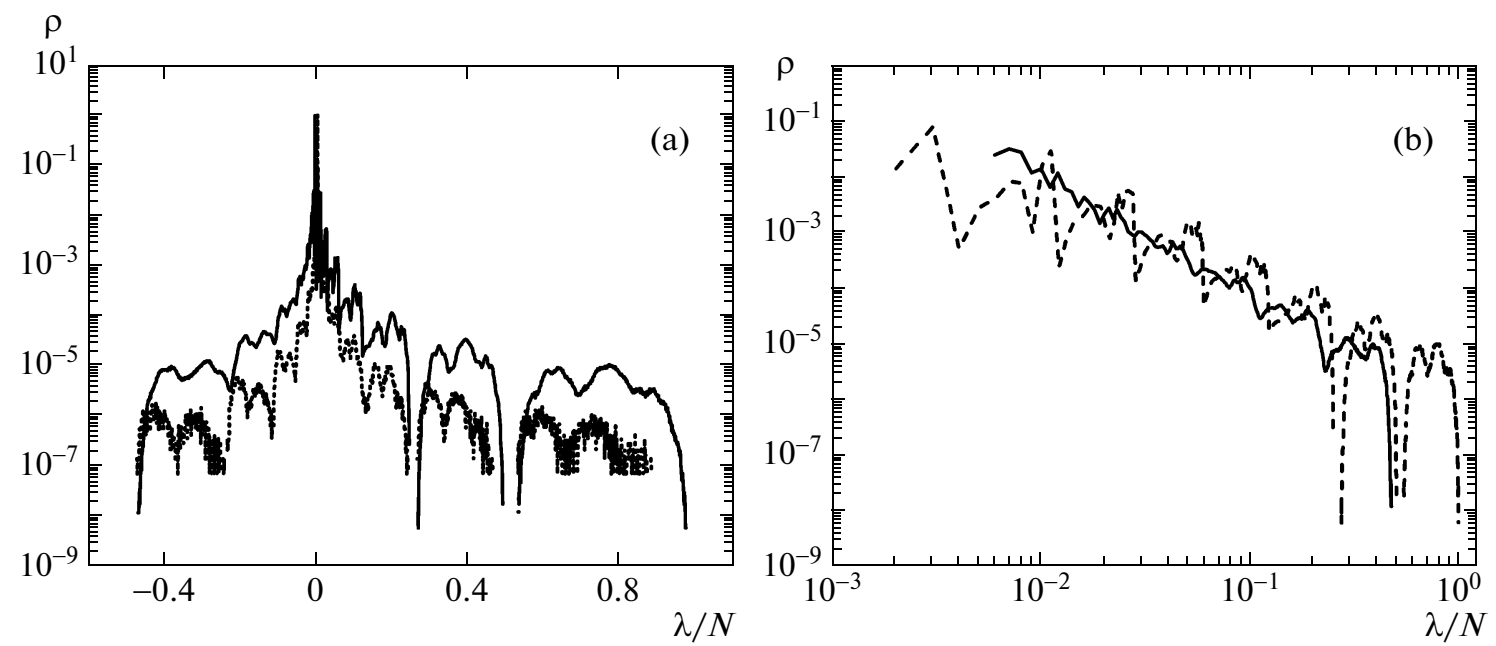

Fig. 4. Spectral density $\rho(\lambda)$ : (a) $\mu=0.2, N=256$ (solid curve), $N=1024$ (dotted curve); (b) left (solid curve) and right (dashed curve) tails of distribution $\rho(\lambda)$ for $N=256$.

probabilities $\{Q\}=\left\{q_{1}, q_{2}, \ldots, q_{\Gamma}\right\}$. Thus, elements $t_{\gamma}^{(n)}$ and the network links corresponding to them (which are random quantities with a binomial distribution) are organized hierarchically in probabilities. We will consider below a set of probabilities $\{Q\}$ with $q_{\gamma}=p^{-\mu \gamma}(\mu>0)$.

Systematic study of statistical properties of random network ensembles primarily necessitates analysis of the spectral properties of the adjacency matrix [39, 40]. Let $\lambda_{i}(1 \leq i \leq N)$ be the eigenvalues of a certain realization of the PAM. Spectral density $\rho(\lambda)$ averaged over the ensemble of random PAMs is defined in the standard way:

$$
\rho(\lambda)=\frac{1}{N} \sum_{i=1}^{N}\left\langle\delta\left(\lambda-\lambda_{i}\right)\right\rangle_{\left\{q_{1}, q_{2}, \ldots, q_{\Gamma}\right\}} .
$$

Evaluating numerically the spectral density $\rho(\lambda)$ for the ensemble of random PAM defined above, we observed [18] that the tails of the $\rho(\lambda)$ distribution clearly demonstrate a power behavior, $\rho(\lambda) \propto|\lambda|^{-\chi}$ with exponent $\chi=\chi(\mu)$. Figure 4 shows by way of example the spectral density $\rho(\lambda)$ (for $N=256,1024$, and $\mu=0.2$ ) in semilogarithmic coordinates; the corresponding tails in the distribution are given in Fig. $4 \mathrm{~b}$ in double logarithmic coordinates.

Let us consider analytic arguments clarifying this observation. We define spectral density $\rho_{G}(\lambda)$ of a random block-hierarchical matrix $T$, assuming that the Bernoulli distribution for elements of matrix $T$ are replaced by Gaussian distributions with zero mean value and with a dispersion set $\{\mathscr{P}\}=\left\{\sigma_{1}, \sigma_{2}, \ldots, \sigma_{\Gamma}\right\}$, where $\sigma_{\gamma}=p^{-v \gamma}(v>0)$. It should be noted that the eigenvalues of any regular (i.e., translation-invariant) block-hierarchical matrix of contacts with zero diagonal elements can be expressed in terms of matrix elements $t_{\gamma}^{(n)} \equiv t_{\gamma}$ analogously to expression (1) for the eigenvalues of a regular PKM (see $[9,12]$ and a generalization made in [20]):

$$
\lambda_{\gamma}=p^{\gamma} t_{\gamma}-\left(1-p^{-1}\right) \sum_{\gamma^{\prime}=1}^{\gamma} p^{\gamma^{\prime}} t_{\gamma^{\prime}} \quad(\gamma=1, \ldots, \Gamma),
$$

where eigenvalue $\lambda_{\gamma}$ is $p^{\Gamma-\gamma}$-fold degenerate $(\gamma=1, \ldots$, $\Gamma)$. Note that, in contrast to the kinetic matrix, eigenvalue $\lambda_{0}=0$ of the matrix of contacts is missing, but eigenvalue $\lambda_{0}=-\sum_{\gamma=1}^{\Gamma} p^{\Gamma-\gamma} t_{\gamma}$ exists. In addition, in spite of the similarity of expressions (1) and (22), we must pay attention to the fact that the summation of matrix elements $t_{\gamma^{\prime}}$ for the eigenvalues of the kinetic matrix is carried out along the path on the tree from vertex $(\gamma, n)$ to the origin, and this path is unique, while summation in expression (22) is carried out from its point $(\gamma, n)$ to the tree boundary (level $\gamma=1)$ (i.e., in the opposite direction), and the number of such summation paths is $p^{\gamma}$. For translation-invariant block-hierarchical matrices, these summation paths are equivalent; consequently, expression (22) is exact both for kinetic matrices and for matrices of contacts. However, generalization (22) for random matrices of contacts cannot be constructed using the same algorithm as that used above for random kinetic matrices. It should be recalled that, for a random PKM (see relation (2)), we have

$$
\lambda_{\gamma, n}=-p^{\gamma} t_{\gamma}^{(n)}-\left(1-p^{-1}\right) \underbrace{\sum_{\gamma^{\prime}=\gamma+1}^{\Gamma} p^{\gamma^{\prime}} t_{\gamma^{\prime}}^{\left(n^{\prime}\right)}}_{\Sigma},
$$

i.e., eigenvalue $\lambda_{\gamma, n}$ can be expressed in terms of a linear combination of matrix elements $t_{\gamma^{\prime}}^{\left(n^{\prime}\right)}$ denoted by $\Sigma$ and corresponding to transitions through vertices $\left(\gamma^{\prime}, n^{\prime}\right)$ along the path from $(\gamma, n)$ to origin $(\Gamma, 1)$ (see Section 2 for details). Using the same geometrical 
interpretation as for the summation path in relation (22), we could try to represent eigenvalue $\lambda_{\gamma, n}$ of the random block-hierarchical adjacency matrix by a linear combination of matrix elements $t_{\gamma^{\prime}}^{\left(n^{\prime}\right)}$ along the path from point $(\gamma, n)$ to the boundary of the $p$-adic tree (see Fig. 1b). Unfortunately, however, such a construction is not defined by point $(\gamma, n)$ alone and depends on the summation path in the translationnoninvariant case. The eigenvalues of specific realizations of a random PAM are parametrized by the paths on the subtree with point $(\gamma, n)$ in contrast to the eigenvalues of a nonrandom PAM, which are parametrized only by point $(\gamma, n)$ of the subtree. Thus, strictly speaking, the exact expression for eigenvalues $\lambda_{\gamma, n}$ cannot be written in the form of linear combination (23) of matrix elements. Nevertheless, the spectral density $\rho_{G}(\lambda)$ averaged over the ensemble of random PAMs can be calculated using a posteriori considerations of self-averaging, which make analysis self-consistent. Note that numerical simulation confirms our analytic prediction of distribution $\rho_{G}(\lambda)$ for $|\lambda| \gg 1$ in the interval $0<v<1$.

Thus, proceeding from the similarity of expressions (1) and (22) and formally using structure (23) for $\lambda_{\gamma, n}$, we replace in relation (23) the direction of the summation path on the tree (i.e., we replace the sum on the right-hand side of relation (22) by $\sum_{\gamma^{\prime}=1}^{\gamma} p^{\gamma^{\prime}} t_{\gamma}^{\left(n^{\prime}\right)}$, where summation is extended along an arbitrarily chosen path from point $(\gamma, n)$ to the tree boundary $\left.\gamma^{\prime}=1\right)$ (cf. relation (23)). Further, assuming that the distribution of matrix elements $t_{\gamma}^{(n)}$ is Gaussian,

$$
P\left(t_{\gamma}^{(n)}\right)=\frac{1}{\sqrt{\pi \sigma_{\gamma}^{2}}} \exp \left(-\frac{\left(t_{\gamma}^{(n)}\right)^{2}}{\sigma_{\gamma}^{2}}\right),
$$

and using for $\lambda_{\gamma, n}$ the expression in the form of a linear combination of matrix elements analogous to (23) but with a modified summation path, we arrive at the following expression for spectral density $\rho_{G}(\lambda)$ :

$$
\begin{gathered}
\rho_{G}(\lambda)=p^{-\Gamma} \sum_{\gamma, n}\left\langle\delta\left(\lambda-\lambda_{\gamma, n}\right)\right\rangle_{P\left(t_{\gamma}^{(n)}\right)} \\
=\frac{1}{\sqrt{\pi}} \sum_{\gamma=1}^{\Gamma} p^{-\gamma} \frac{1}{\sqrt{u_{\gamma}^{2}}} \exp \left(-\frac{\lambda^{2}}{u_{\gamma}^{2}}\right),
\end{gathered}
$$

where we have

$$
\begin{array}{r}
u_{\gamma}^{2}=p^{2(\gamma-1)} \sigma_{\gamma}^{2}+\left(1-p^{-1}\right) \\
\times \sum_{\gamma^{\prime}=1}^{\gamma-1} p^{2 \gamma^{\prime}} \sigma_{\gamma^{\prime}}^{2}=\frac{p-2}{p} p^{2(1-v) \gamma} \\
+\frac{(p-1)^{2}}{p-p^{v}}\left(p^{2(1-v) \gamma}-1\right)
\end{array}
$$

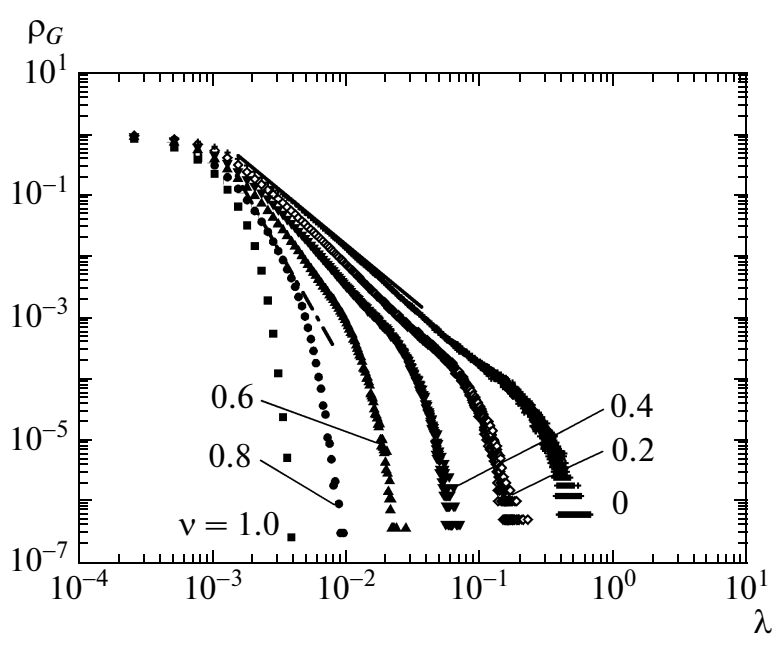

Fig. 5. Spectral density of random (Gaussian) translationinvariant block-hierarchical matrix of contacts. Solid and dot-and-dash curves have slopes of $\xi(v=0)=2$ and $\xi(v=$ $0.8)=6$, respectively; symbols: $v=0(+), 0.2(\diamond), 0.4(\boldsymbol{\nabla})$, $0.6(\boldsymbol{\Delta}), 0.8(\bullet)$, and $1.0(\mathbf{\square})$.

for $\sigma_{\gamma}=p^{-v \gamma}$. Setting $p=2$, we can write the expression for $\rho_{G}(\lambda)(25)$ for $\Gamma \longrightarrow \infty$ in the form

$$
\begin{aligned}
& \rho_{G}(\lambda) \approx \frac{1}{\sqrt{\pi}} \sum_{\gamma=1}^{\infty} 2^{-(2-v) \gamma} \\
& \times \exp \left[-\lambda^{2} \frac{4-4^{v}}{2^{2(1-v) \gamma}-1}\right] .
\end{aligned}
$$

Considering that

$$
\sum_{\gamma=1}^{\infty} p^{-c_{1} \gamma} \exp \left(-t p^{-c_{2} \gamma}\right) \approx t^{-c_{1} / c_{2}} \quad(t \gg 1),
$$

where $c_{1}=2-v$ and $c_{2}=1-v$, we obtain the following asymptotic form of the spectral density for $|\lambda| \gg 1$ :

$$
\rho_{G}(\lambda) \approx|\lambda|^{-\xi(v)} \quad(0<v<1),
$$

where

$$
\xi(v)=(2-v) /(1-v) .
$$

The considerations justifying our derivation of expression (25) for spectral density $\rho_{G}(\lambda)$ are as follows. It should be noted above all that expression (25) becomes exact if random matrix elements $t_{\gamma}^{(n)}$ become degenerate in $n$ (which restores translation invariance of block-hierarchical matrix $T$ ). Second, the results of numerical simulation presented in Fig. 5 show that relations (29) and (30) indeed hold for random blockhierarchical adjacency matrices. Figure 5 illustrates the behavior of spectral density $\rho_{G}(\lambda)$ for Gaussian random block-hierarchical matrices of size 256.

A regular asymptotic form of spectral density $\rho_{G}(\lambda)$ for $|\lambda| \gg 1$ obviously emerges owing to effective selfaveraging of sum $\sum_{\gamma^{\prime}=1}^{\gamma} p^{\gamma^{\prime}} t_{\gamma}^{\left(n^{\prime}\right)}$ of the matrix element 
along each specific path (from the given point to the tree boundary) for matrices defined by distributions $\{\mathscr{P}\}=\left\{\sigma_{1}, \sigma_{2}, \ldots\right\}$. It can easily be seen that the lower summation limit in the sum in expression (26) for $\sigma_{\gamma}=$ $p^{-v \gamma}$ can be shifted from $\gamma^{\prime}=1$ to $\gamma^{\prime} \longrightarrow-\infty$. The result for $u_{\gamma}^{2}$ remains asymptotically the same if $0<v<1$. Such an extension of the summation interval indicates that the evaluation of spectral density (and, in particular, $\lambda_{\gamma, n}$ ) involves summation along infinitely long paths extending from $-\infty$ up to hierarchical level $\gamma$. We can expect that, for $\sigma_{\gamma}=p^{-v \gamma}(0<v<1)$, the contribution to eigenvalue $\lambda_{\gamma, n}$ is independent of specific summation paths leading from the point of level $\gamma$ to $-\infty$ in view of the convergence of sum $\sum_{\gamma^{\prime}=-\infty}^{\gamma} p^{2 \gamma^{\prime}} \sigma_{\gamma^{\prime}}^{2}$; i.e., effective eigenvalues are degenerate in index $n$. As a result, our analysis boils down to a translation-invariant PAM for which expression (25) is exact.

It can easily be seen from formula (29) and Fig. 5 that, for $v \geq 1$, the power behavior of spectral density $\rho_{G}(\lambda)$ is not observed. This fact deserves special discussion. Returning to expression (26), we see that the lower limit of summation over $\gamma^{\prime}$ for $v \geq 1$ cannot be shifted to $-\infty$ since the corresponding sum in this case will diverge. Thus, the contribution to the eigenvalue (and, hence, to spectral density) for $v \geq 1$ strongly depends on the configuration of a specific path on the Cayley tree. In this case, we cannot use considerations concerning self-averaging of spectral density; we cannot reliably determine as yet the behavior of $\rho_{G}(\lambda)$ for $v \geq 1$.

Concluding this section, we note that our approach to constructing a "heavy tail" (i.e., power dependence) in the spectral density of ensembles of random graphs is not unique. Recent publication [41] is worth mentioning in this connection, in which it is proposed that Gaussian random matrices should be divided into random fragments with a certain preset distribution function. The application of such a procedure to random graphs led in [42] to interpolation of spectral density between the corresponding expressions for scale-free and completely random networks.

\subsection{Hierarchical Growth of Polyscale Random Networks}

Two basically different approaches to constructing a scale-free network can be distinguished. One of these methods, which was mainly proposed for purposes of illustration, involves the construction of a hierarchical graph with certain fractal properties [42]. Since graphs are deterministic in this case, it is meaningless to speak about their statistics. Another widely used method deals with various versions of iterative construction of a random network with preferential attachment of nodes [43], in which new nodes are added to the nodes of the network with a probability that depends on the existing vertex degree (number of direct links between the given node and other nodes). Almost all known statistical characteristics of scale-free networks, including the spectral density of the adjacency matrix, were obtained for networks constructed by this method. The corresponding spectral density has a typical form of a triangular distribution in the bulk with tails decreasing in accordance with a power law. It should be noted that the construction of a scale-free network using the preferential attachment method is based on a locally nonuniform procedure of growing a network with unlimited evolution memory.

We propose, however, a different approach to constructing a random block-hierarchical network with a power behavior of spectral density, which is based on a uniform and parallel (rather than step-by-step) procedure. Namely, we construct random scale-free networks in accordance with the adjacency matrix obtained by appropriate randomization of a blockhierarchical matrix of the Parisi type.

The peculiarity and novelty of our construction is that we construct clusters of links with hierarchically organized probabilities, while the typical procedure is based on hierarchical grouping of vertices of a random graph (network). This allows us to construct a hierarchical network by local uniform growing without evolution memory. Our network growing procedure does not presume any metric structure of the graph (network) and leaves the graph purely topological. Conversely, the traditional grouping of vertices implies the presence of a metric on the set of graph (network) vertices since such a grouping is usually based on concept of "close" (or "distant") vertices.

For better understanding of the spectral properties of random hierarchical graphs, we can use the relation between the theory of random matrices and the theory of random graphs (networks). The spectral density of an ensemble of random symmetric adjacency matrices,

$$
\rho(\lambda)=\frac{1}{N} \sum_{i=1}^{N}\left\langle\delta\left(\lambda-\lambda_{i}\right)\right\rangle_{\left\{q_{1}, q_{2}, \ldots, q_{\Gamma}\right\}},
$$

is directly related to the topological structure of the graph since the quantity

$$
M_{k}=\frac{1}{N} \int \lambda^{k} \rho(\lambda) d \lambda=\frac{1}{N} \sum_{i=1}^{N} \lambda_{i}^{k}
$$

defines (to within a factor of $N$ ) the mean number of $k$-step loops on a random graph (see, for example, [39]). In particular, it was shown [44] that the spectral properties of random ER graphs [25] in the thermodynamic limit $(N \longrightarrow \infty)$ coincide with the spectral properties of random real-valued symmetric matrices. This result is the starting point of our analysis. It should be recalled that elements $A_{i, j}$ of the adjacency matrix of an ER graph are random quantities from the Bernoulli distribution: $A_{i, j}=1$ or $A_{i, j}=0$ with probability $q$ or $1-q$, respectively. 

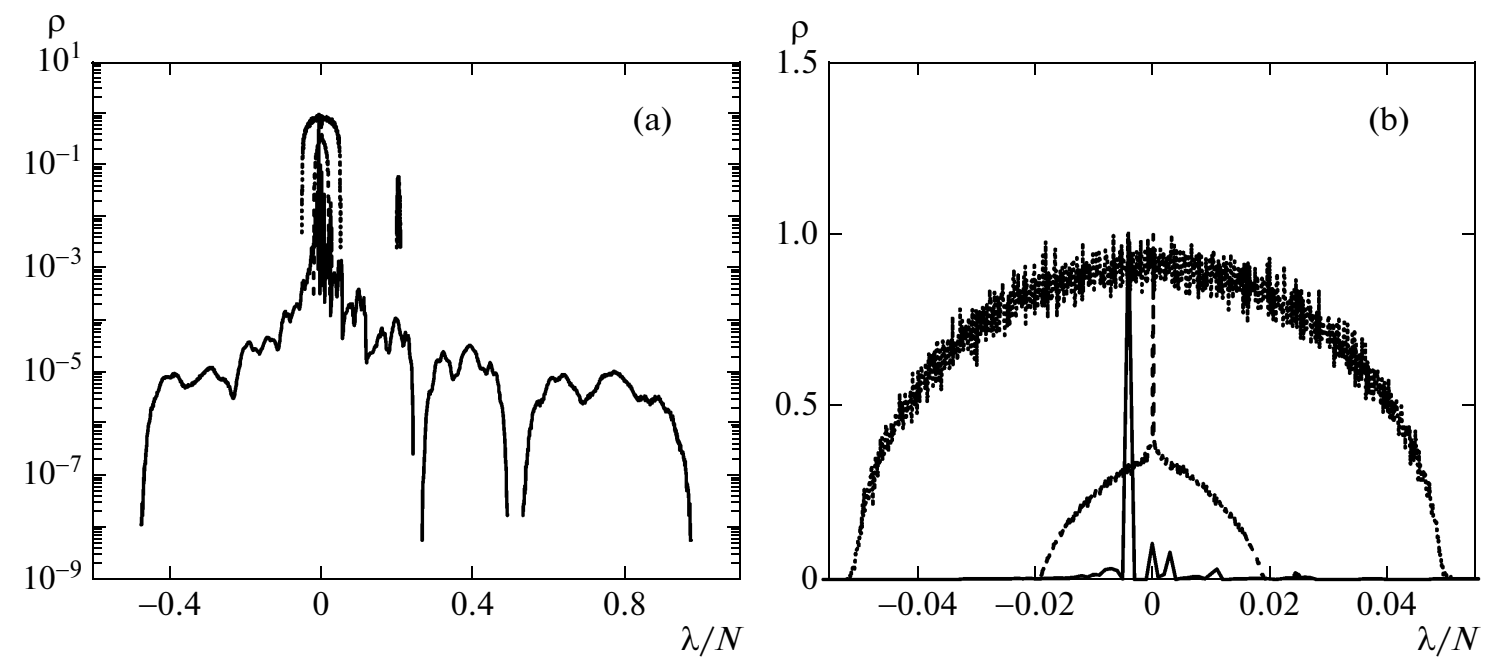

Fig. 6. Comparison of spectral densities of adjacency matrices of random hierarchical networks (solid curves, $\mu=0.2$ ) and random ER graphs ( $p=0.2$, dotted curves; $p=0.02$, dashed curves) for $N=256$ in (a) semilogarithmic and (b) linear coordinates.

For an ensemble of random ER graphs, density distribution $\rho_{A}(\lambda)$ of the eigenvalues of adjacency matrix $A$ can be calculated analytically in thermodynamic limit $(N \longrightarrow \infty)$ and has the form of the WignerDyson semicircle law [45] observed for Gaussian random matrices. Namely, the following statement was proved in [44]. Let $B$ be a real $N \times N$ symmetric matrix with independent matrix elements $B_{i, j}$ from, say, Gaussian distribution $P\left(B_{i, j}\right)$ with $\left\langle B_{i, j}\right\rangle=0$ and $\left\langle B_{i, j}^{2}\right\rangle=\sigma^{2}$. Then the spectral density $\rho_{B}(\lambda)$ of the ensemble of matrices $B$ in the limit $N \longrightarrow \infty$ asymptotically tends to the semicircle distribution law:

$$
\rho_{B}(\lambda)= \begin{cases}\frac{1}{2 \pi \sigma^{2}} \sqrt{4 N \sigma^{2}-\lambda^{2}}, & |\lambda|<\sqrt{4 N \sigma^{2}} \\ 0, & |\lambda|>\sqrt{4 N \sigma^{2}} .\end{cases}
$$

If $\sigma^{2}=q(1-q)$, then $\rho_{A}(\lambda)=\rho_{B}(\lambda)$ for $N \longrightarrow \infty$; i.e., the spectral densities of ensembles of random ER graphs and Gaussian symmetric real matrices coincide in the thermodynamic limit. At the same time, such a coincidence of spectral densities of random graphs and random Gaussian matrices should not be treated literally since some spectral properties of ER graphs differ from those of Gaussian matrices [39, 46]. For example, since $\left\langle A_{i, j}\right\rangle=q$ for the adjacency matrix of an ER graph, the corresponding largest eigenvalue $\lambda_{1}$ increases linearly with size $N$ of the system (i.e., $\lambda_{1}=$ $N q$ ). This means that the semicircle law for a random ER graph distribution is valid only for matrix $A-\langle A\rangle$. In addition, the tails of the spectral density distributions near the spectrum edge for ER graphs and Gaussian matrices are different. Nevertheless, the similarity of statistical properties of random ER graphs and ran- dom Gaussian matrices in the thermodynamic limit may serve as the starting point for analysis of statistical properties of hierarchical random graphs. The spectral properties of hierarchical random graphs and ER graphs are compared in Fig. 6.

Figure 7 shows the results of numerical simulation of probability distribution $P\left(M_{k}\right)(k=2,3)$ for $k$-step loops in random hierarchical graphs for $\mu=0.2$. The distribution of loops on ER graphs for $N=256$ and $p=$ 0.5 is also shown for comparison. It should be recalled that $P\left(M_{k}\right)$ is the probability that the mean connectivity on a finite graph is $M_{k}$.

It can be seen that distribution functions $P\left(M_{2}\right)$ and $P\left(M_{3}\right)$ for the random graphs represented by blockhierarchical adjacency matrices are much broader than the corresponding distributions for ER graphs with the same number of vertices; consequently, it is natural to refer to the corresponding structure of a random hierarchical graph as a polyscale structure.

Using the generating function method, we can easily calculate the vertex degree distribution in an ensemble of random block-hierarchical graphs directly from their connectivity matrices. For this purpose, we consider an arbitrary (e.g., first) row of adjacency matrix $T$ (see Fig. 3b). The total number of links of the first graph vertex with the remaining vertices is defined by the number of nonzero matrix elements $t_{\gamma}^{(1)}$ $(\gamma=1, \ldots, \Gamma)$ in the first row (i.e., the number of matrix elements equal to unity). Thus, the vertex degree distribution $\mathscr{P}(m)$ is probability $\mathscr{P}$ that the sum of matrix elements in the first row is exactly equal to $m$ provided that the matrix elements are grouped into hierarchical blocks and obey binomial distributions $\left\{q_{1}, q_{2}, \ldots, q_{\Gamma}\right\}$ (see relation (20)). As a result, we obtain the following 

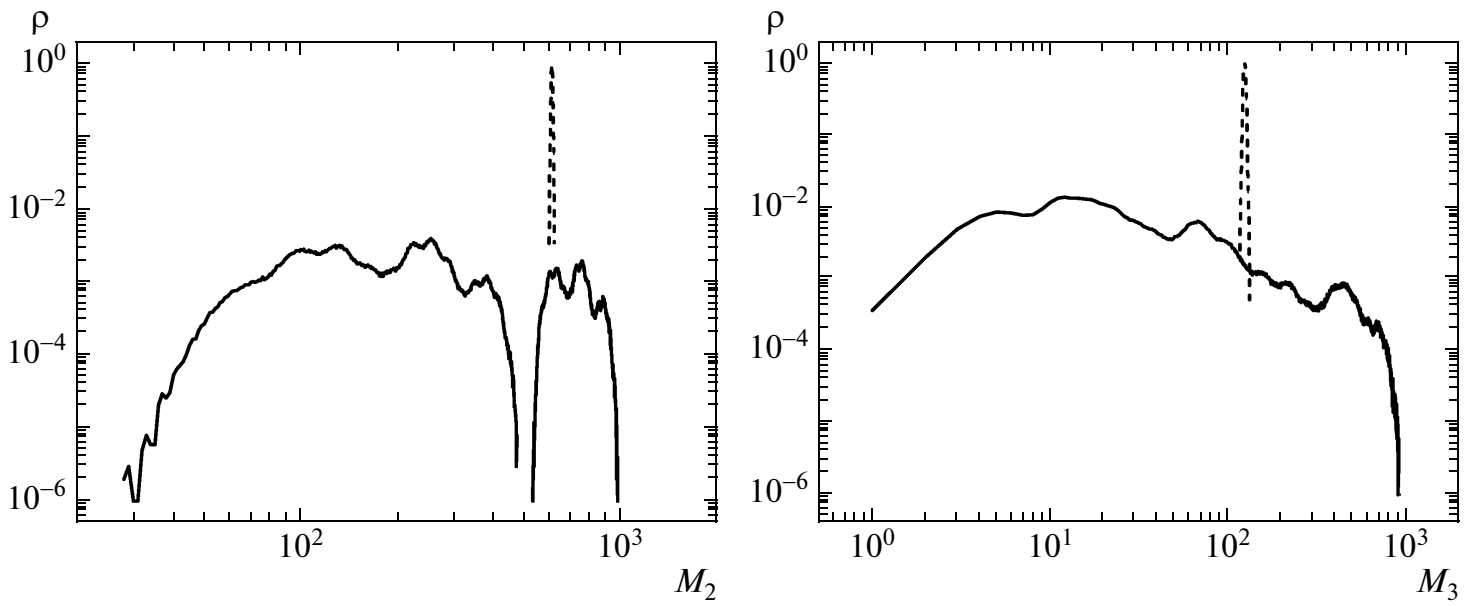

Fig. 7. Distributions of loops in random hierarchical graphs (solid curves) and random ER graphs (dashed curves).
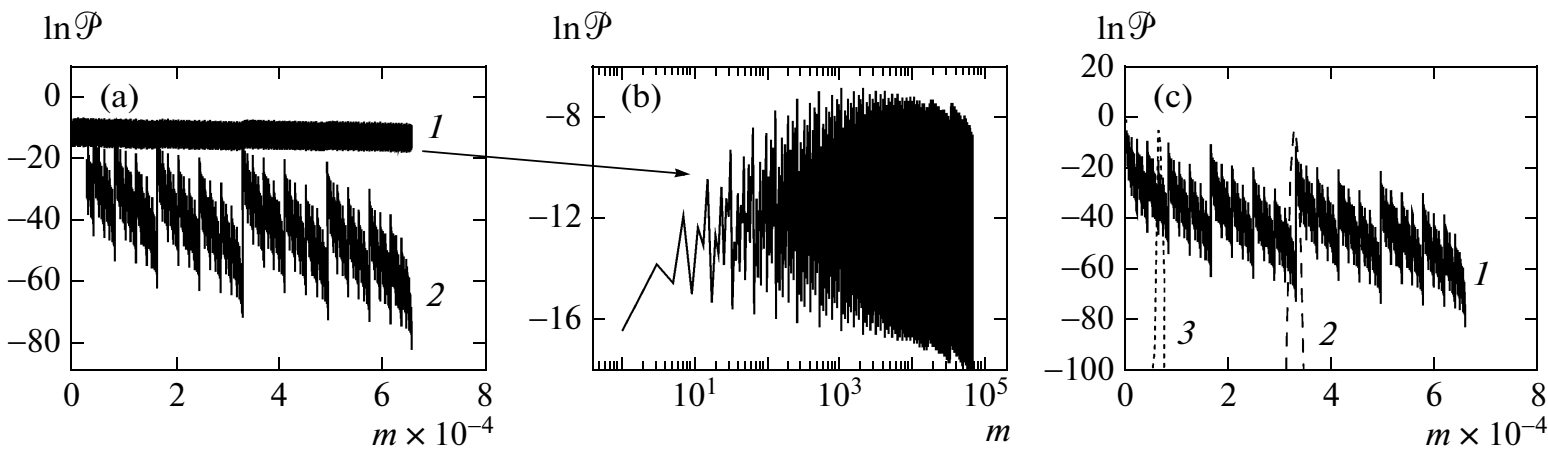

Fig. 8. (a) Family of distributions $\mathscr{P}(m)$ at $\Gamma=16$ for $\mu=0.1(1)$ and $1.0(2)$; (b) density of distribution $\mathscr{P}(m)$ on double logarithmic scale for $\mu=0.1$ and $\Gamma=16$; (c) comparison of $\mathscr{P}(\mathrm{m})$ for a random block-hierarchical graph $(\mu=1.0, \Gamma=16$, curve 1$)$ and $\mathscr{P}_{E R}(m)$ for an ER graph with $N=2^{16}$ and $q=0.1(2), 0.5$ (3).

expression for $\mathscr{P}(m)$ (to simplify notation, we set where $\left.q_{\gamma}\left(t_{\gamma}^{(1)}\right) \equiv q_{\gamma}\left(t_{\gamma}\right)\right)$ :

$$
\mathscr{P}(m)=\sum_{\left\{t_{1} \ldots t_{\Gamma}\right\}}\left[\prod_{\gamma=1}^{\Gamma} q_{\gamma}\left(t_{\gamma}\right)\right] \Delta\left(\sum_{\gamma=0}^{\Gamma} p^{\gamma} t_{\gamma+1}-m\right),
$$

where binomial distributions $q_{\gamma}\left(t_{\gamma}\right)$ have the form

$$
q_{\gamma}\left(t_{\gamma}\right)=p^{-\mu \gamma} \delta_{q_{\gamma}\left(t_{\gamma}\right), 1}+\left(1-p^{-\mu \gamma}\right) \delta_{q_{\gamma}\left(t_{\gamma}\right), 0},
$$

and $\Delta(\ldots)$ is the Kronecker delta,

$$
\Delta(x)=\frac{1}{2 \pi i} \oint d z z^{x-1}= \begin{cases}1, & x=0 \\ 0, & x \neq 0 .\end{cases}
$$

Substituting expressions (34) and (35) into (33) and performing elementary transformations, we obtain

$$
\mathscr{P}(m)=\frac{1}{2 \pi i} \oint d z z^{-(m+1)} \prod_{\gamma=1}^{\Gamma} W(z, \Gamma),
$$

$$
W(z, \Gamma)=p^{-\mu \gamma} z^{p^{\gamma}}+1-p^{-\mu \gamma} .
$$

For finite but not very large values of $\Gamma$, the expression for distribution function $\mathscr{P}(m)$ can be analyzed numerically. Taking advantage of the fact that function $W(z, \Gamma)$ is a polynomial in $z$, we can write $\mathscr{P}(m)$ in the form

$$
\mathscr{P}(m)=\left.\frac{1}{(m+1) !} \frac{d^{m+1} \prod_{\gamma=1}^{\Gamma} W(z, \Gamma)}{d z^{m+1}}\right|_{z=0} .
$$

Figure 8 a shows the family of curves $\mathscr{P}(m)$ for $\Gamma=16$ for values of $\mu=0.1$ and 1.0. Figure $8 \mathrm{c}$ shows for comparison the distributions $\mathscr{P}(m)$ for $\mu=1.0$ and $\Gamma=16$ as well as the binomial distribution $\mathscr{P}_{E R}(m)=$ 
$C_{N}^{m} q^{m}(1-q)^{N-m}$ for standard ER graphs with number of vertices $N=2^{\Gamma}=2^{16}$ and for $q=0.1,0.5$. It can be seen that distribution $\mathscr{P}(m)$ of the number of links in a random block-hierarchical network is broader than the corresponding distributions for random ER networks.

The fractal structure of the $\mathscr{P}(m)$ distribution for random hierarchical networks, which is clearly manifested in Figs. 8a and 8b, is apparently closely related to invariant multifractal measures emerging in random Hamiltonian systems in analysis of a number of problems of number-theoretic chaos (see, for example, [47]). Indeed, the condition $\Delta\left(\sum_{\gamma=1}^{\Gamma} p^{\gamma} t_{\gamma}-m\right)$ in expression (33) for $\Gamma \longrightarrow \infty$ is precisely the binary expansion of number $m$,

$$
m=t_{1} 2^{0}+t_{2} 2^{1}+t_{3} 2^{2}+\ldots+t_{\gamma+1} 2^{\gamma}+\ldots
$$

where coefficients $t_{\gamma}$ assume values of 1 or 0 with corresponding probabilities $q_{\gamma}\left(t_{\gamma}\right)$ defined in relation (34). It should be noted that a similar expansion of the type $\sum_{k=1}^{\infty} \varepsilon_{k} u^{-k}$, where $u>1$ and $\varepsilon_{k}$ assumes values of \pm 1 (with the same probability of $1 / 2$ irrespective of $k$ ), is known in the literature as the singular Erdös measure [48-50]. The observed fractal structure of distribution function $\mathscr{P}(m)$ emerges owing to incommensurability effects of number-theoretic origin: some values of $m$ permit a large number of different expansions in the form of a binary sequence with random coefficients, while the number of such expansions for other values of $m$ is much smaller.

\section{CONCLUSIONS}

It should be noted above all that the observed relationship between relaxation kinetics on randomized ultrametric landscapes and directed polymers on trees with disorder may prove very effective for establishing a deeper correspondence between $p$-adic stochastic models and models with disorder on tree-like structures. We believe that the analysis in Section 2 is a certain advancement in this direction.

Analysis of the properties of random hierarchical graphs in Section 3 is far from being complete; many other properties (e.g., eigenvectors and inverse participation ratio) should also be considered (see, for example, [40]). The main goal of our discussion was to demonstrate the fundamental difference between statistical properties of random graphs constructed in the block-hierarchical manner and completely random ER graphs. The power behavior of the tails in the spec- tral density of adjacency matrices of block-hierarchical graphs allows us to attribute hierarchical graphs (networks) considered here to the class of scale-free graphs, while these networks can be classified as polyscale in accordance with the topological difference from ER graphs.

In conclusion, we would like to emphasize once again the similarity and difference in the two methods for constructing scale-free networks. The main features of these approaches are as follows.

(i) The first method widely discussed in the literature is mainly based on the preferential attachment procedure, in which the network is grown using an essentially non-Markovian (in increments) evolutionary process with unlimited memory. In a certain sense, such evolution requires the control of the entire structure of the network at each step since the formation of new links depends on the current vertex degree distribution over all points of the graph (network). From this point of view, consecutive growth of a scale-free network can be conditionally referred to as nonlinear evolution.

(ii) We have considered here an essentially different mechanism of the formation of a scale-free network. The hierarchical organization of the probability of link cluster formation in a topological network constitutes the main idea of our construction, which is essentially Markovian.

Summing up, we should emphasize that there exist two important properties of hierarchical scale-free networks constructed here. First of all, any subgraph belonging to a certain hierarchical level $\gamma$ is a completely random graph since the formation of a cluster of links (subgraph) at each hierarchical level is completely uncorrelated. Further, random subgraphs associated with various hierarchical levels of link organization in the network may be different, so that the network is generally inhomogeneous as a whole. Nevertheless, it was shown above that successive embeddings of subgraphs corresponding to different hierarchical levels in a very broad range of generation probabilities $\{Q\}=\left\{q_{1}, q_{2}, \ldots\right\}$ lead (depending on statistical characteristics being studied) to a scale-free (or polyscale) network structure. This observation is rather unexpected since the scale-free construction is obtained in our case using an essentially Markovian procedure of growing a network without evolution memory. This suggests that random scale-free hierarchical networks might be encountered among natural networks with origins that do not envisage the condi- 
tions for essentially correlated evolutionary events. In particular, scale-free hierarchical networks (e.g., of chemical origin) may be of interest for prebiotic problems involving the formation and development of the primary forms of the hierarchical structural and functional organization of the biological type.

\section{ACKNOWLEDGMENTS}

The authors are grateful to K.V. Bashevoi for his assistance in computer calculations, O. Bohigas and Ya.V. Fedorov for fruitful discussions at various stages of the research, and M. Mezard for valuable remarks concerning the relation between random block-hierarchical kinetic matrices and the statistics of directed polymers on random trees.

This study was partly financed by the Russian Foundation for Basic Research (project no. 07-0200612a) and program no. 15 of the Presidium of the Russian Academy of Sciences.

\section{APPENDIX}

Historically, the eigenvectors and eigenvalues of the Parisi kinetic matrix (PKM) were calculated for the first time in [9] for a special type of PKMs corresponding to a ultrametric random walk over a regular 2-adic tree. Later, it was proved in [12] that the PKM spectrum determined in [9] coincides with the spectrum of the translation-invariant $p$-adic pseudodifferential Vladimirov operator [6] (see expression (1)). Subsequently, a method for calculating the spectra of $p$-adic pseudodifferential operators of a quite general form was constructed in $[20,51]$; thus, the initial construction from [9] was generalized for translational-noninvariant symmetric PKMs also (see expression (2)). The main elements of this construction will be explained below using the matrix representation.

In the models of ultrametric random walk, PKMs appear in a natural way, and the structure of eigenvectors of PKMs directly reflects the construction of this random process. To describe the eigenvectors of a PKM, let us first construct an ultrametric space.

We consider a set of points $B_{p}(\Gamma)$ labeled by index $i=1, \ldots, N$, where $N=p^{\Gamma}$ and $p \geq 2$. We introduce the ultrametric space on $B_{p}(\Gamma)$. For this purpose, we construct a regularly branching $p$-adic graph $G_{p}(\Gamma)$ (Cayley tree with ramification index $p+1)$, in which boundary nodes form a set $B_{p}(\Gamma)$. Thus, graph $G_{p}(\Gamma)$ has $\Gamma$ hierarchical levels by construction. We assume that distance $d(i, j)$ between any two points $i, j \in B_{p}(\Gamma)$ is defined by the vertex of the smallest subtree (subgraph) $G_{p}\left(\gamma, n_{\gamma}\right) \subseteq G_{p}(\Gamma)$ with boundary nodes including points $i$ and $j$. In our notation, $1 \leq \gamma \leq \Gamma$ indicates the number of the level on tree $G(\Gamma)$ on which the ver- tex of subgraph $G_{p}\left(\gamma, n_{\gamma}\right)$ is located, while $1 \leq n_{\gamma} \leq p^{\Gamma-\gamma}$ is the number of the required vertex among $p^{\Gamma-\gamma}$ vertices located at level $\gamma$. Obviously, the distance introduced in this way on $B_{p}(\Gamma)$ satisfies the strong triangle inequality $d(i, j) \leq \max \{d(i, k), d(j, k)\}$; i.e., set $B_{p}(\Gamma)$ is an ultrametric space by construction. Note that, on a regular $p$-adic graph $G_{p}(\Gamma)$, all subgraphs with vertices lying at the same branching level in $G_{p}(\Gamma)$ are topologically identical. Translation invariance of the ultrametric space has precisely this meaning. Finally, since the strong triangle inequality governs the so-called $p$ adic numerical norm (where $p$ is a prime number), an ultrametric space is described by $p$-adic numbers.

A random walk over an ultrametric space is a uniform Markovian random process with all features essentially determined by the geometry of the ultrametric space. The main feature of the ultrametric space is that it is generated by a regular $p$-adic tree and is naturally associated with the hierarchy of embeddings of smaller spatial regions into larger regions in accordance with the hierarchical structure of embedding of subgraphs $G_{p}\left(\gamma, n_{\gamma}\right)$ into $G_{p}(\Gamma)$. The hierarchy of embeddings can easily be seen in Fig. 1b. Indeed, the set of points $B_{p}(\Gamma)$ is a union of $p$ nonintersecting subsets over $p^{\Gamma-1}$ points in each subset ( $p=2$ in Fig. 1b). These subsets correspond to $p$ subtrees with points lying at level $\Gamma-1$. The ultrametric distance between points $i, j \in B_{p}(\Gamma)$ belonging to different subsets $B_{p}\left(\Gamma-1, n_{\Gamma-1}\right)$ is defined by vertex $(\Gamma, 1)$ (origin of the tree): it is convenient to define this distance as $p^{\Gamma}$ in accordance with the definition of the $p$-adic numerical norm. Each subset of level $\Gamma-1$ can in turn be treated as a union of $p$ nonintersecting subsets of level $\Gamma-2$ over $p^{\Gamma-2}$ states in each subset. The distance between the points belonging to different subsets of level $\gamma=\Gamma-2$ depends on the level at which these subsets merge into one. If this occurs at the next level $\Gamma-1$, the distance between these points is $p^{\Gamma-1}$; if, however, $B_{p}(\Gamma)$ is the common set, the distance between such points is $p^{\Gamma}$. It should be recalled that we consider only the points belonging to different subsets of level $(\Gamma-2)$. To determine the distance between points belonging to the same subset of level $\Gamma-2$, we must further divide these subsets into smaller subsets embedded in them up to level $\gamma=1$. Such divisions correspond to hierarchy of distances $\left\{p^{1}, p^{2}, \ldots, p^{\Gamma}\right\}$ on space $B_{p}(\Gamma)$.

An ultrametric random walk over $B_{p}(\Gamma)$ is a uniform Markovian process with a transition matrix defined by the following construction. We fix a pair of points $i$ and $j$ from $B_{p}(\Gamma)$. For this pair of points, we find on $p$-adic graph $G_{p}(\Gamma)$ the smallest subgraph containing points $i$ and $j$. Such a subgraph is unique and defines the minimal subset in which a (symmetric) transition $i \longleftrightarrow j$ takes place. Let this subgraph correspond to a vertex 
with indices $\left(\gamma, n_{\gamma}\right)$. On this subgraph, we choose two subgraphs of a lower lying level with vertices $(\gamma-1$, $\left.n_{\gamma-1}\right)$ and $\left(\gamma-1, n_{\gamma-1}^{\prime}\right)$, which are embedded in it. Note that points $i$ and $j$ obviously belong to different subgraphs (subsets) of level $\gamma-1$. By construction, the probability of transition $i \longleftrightarrow j$ (per unit time) of ultrametric random walk is completely determined by a pair of the largest subsets between which the transition takes place. In other words, elements $T_{i, j}$ of the transition matrix are parametrized by three indices: pair $\left(\gamma, n_{\gamma}\right)$ defining the smallest subset in the embedding hierarchy, in which the transition occurs, and an additional index $k(1 \leq k \leq p-1)$ that fixes a pair of the largest subsets embedded in it, between which the transition takes place. Therefore, the geometry of the ultrametric space generates the block-hierarchical structure of the transition matrix (i.e., the PKM structure). Note that the total number of triples $\left(\gamma, n_{\gamma}, k\right)$ parametrizing the vertices of the subgraphs of the $\Gamma$-level $p$-adic graph $\left(p^{\Gamma}-1\right)$ coincides with the number of nonzero eigenvalues of the $p^{\Gamma} \times p^{\Gamma}$ PKM.

The eigenvector of the PKM is the column vector with $p^{\Gamma}$ complex (in the general case) components, which reflects in a certain sense the structure of basins of the ultrametric space described above and the construction of the ultrametric random walk. The eigenvectors of the PKM are parametrized by triples $\left(\gamma, n_{\gamma}, k\right)$. Each level $\gamma$ is juxtaposed to $(p-1) p^{\gamma}$ eigenvectors $\mathbf{e}_{p}\left(\gamma, n_{\gamma}, k\right)$ in accordance with the number of triples $\left(\gamma, n_{\gamma}, k\right)$ with fixed $\gamma$. The components of the eigenvector corresponding to level $\gamma$ form $p^{\Gamma-\gamma}$ groups with $p^{\gamma}$ elements in each. Only one of such groups has nonzero components. The group of nonzero components consists of $p$ subgroups with $p^{\gamma-1}$ identical elements in each subgroup. The components of one of such subgroup are equal to unity, while components of the remaining $p-1$ subgroups are complex numbers of the form $\exp \left[2 \pi i a\left(\gamma, n_{\gamma}, k\right)\right]$, which are selected so that the sum of all nonzero vector components is zero. Thus, triple $\left(\gamma, n_{\gamma}, k\right)$ completely defines the structure of the eigenvector of the PKM (i.e., the number of nonzero components of the column vector, the location of the group of nonzero components in the column, and the structure of the subgroups of this group).

Let us illustrate the structure of eigenvectors of PKMs by two simplest examples. We take a 2-adic graph corresponding to an $8 \times 8$ PKM $(\Gamma=3)$. Level $\gamma=1$ is juxtaposed to four eigenvectors:

$$
\begin{aligned}
& \mathbf{e}_{2}(1,1)=\left(\begin{array}{c}
1 \\
-1 \\
0 \\
0 \\
0 \\
0 \\
0 \\
0
\end{array}\right), \mathbf{e}_{2}(1,2)=\left(\begin{array}{c}
0 \\
0 \\
1 \\
-1 \\
0 \\
0 \\
0 \\
0
\end{array}\right), \\
& \mathbf{e}_{2}(1,3)=\left(\begin{array}{c} 
\\
0 \\
0 \\
0 \\
0 \\
1 \\
-1 \\
0 \\
0
\end{array}\right), \mathbf{e}_{2}(1,4)=\left(\begin{array}{c}
0 \\
0 \\
0 \\
0 \\
1 \\
-1
\end{array}\right) .
\end{aligned}
$$

Level $\gamma=2$ is juxtaposed to two eigenvectors:

$$
\mathbf{e}_{2}(2,1)=\left(\begin{array}{c}
1 \\
1 \\
-1 \\
-1 \\
0 \\
0 \\
0 \\
0
\end{array}\right), \quad \mathbf{e}_{2}(2,2)=\left(\begin{array}{c}
0 \\
0 \\
0 \\
0 \\
1 \\
1 \\
-1 \\
-1
\end{array}\right) .
$$

Finally, the root vertex of the graph is juxtaposed to single eigenvector

$$
\mathbf{e}_{2}(3,1)=\left(\begin{array}{c}
1 \\
1 \\
1 \\
1 \\
-1 \\
-1 \\
-1 \\
-1
\end{array}\right) \text {. }
$$

Note that the blocks of a 2-adic PKM are labeled only by pairs $(\gamma, n)$. It can easily be verified by direct substitution that each of the above vectors is an eigenvector of the 2-adic PKM. Supplementing the set of seven 
given vectors by an additional unit vector $\mathbf{e}_{0}$, we obtain the complete basis of 2-adic symmetric $8 \times 8$ PKMs.

Let us write the explicit expressions for eigenvalues of our PKM:

$$
\begin{aligned}
& \lambda_{1,1}=\varepsilon^{(1)}-q_{1}^{(1)} \\
& =-2 q_{1}^{(1)}-\left(1-2^{-1}\right) \sum_{\gamma^{\prime}=2}^{3} 2^{\gamma^{\prime}} q_{\gamma^{\prime}}^{(1)} \text {, } \\
& \lambda_{1,2}=\varepsilon^{(2)}-q_{1}^{(2)} \\
& =-2 q_{1}^{(2)}-\left(1-2^{-1}\right) \sum_{\gamma^{\prime}=2}^{3} 2^{\gamma^{\prime}} q_{\gamma^{\prime}}^{(2)} \text {, } \\
& \lambda_{1,3}=\varepsilon^{(3)}-q_{1}^{(3)} \\
& =-2 q_{1}^{(3)}-\left(1-2^{-1}\right) \sum_{\gamma^{\prime}=2}^{3} 2^{\gamma^{\prime}} q_{\gamma^{\prime}}^{(3)} \text {, } \\
& \lambda_{1,4}=\varepsilon^{(4)}-q_{1}^{(4)} \\
& =-2 q_{1}^{(4)}-\left(1-2^{-1}\right) \sum_{\gamma^{\prime}=2}^{3} 2^{\gamma^{\prime}} q_{\gamma^{\prime}}^{(4)}, \\
& \lambda_{2,1}=\varepsilon^{(1)}+q_{1}^{(1)}-2 q_{2}^{(1)}=\varepsilon^{(2)}+q_{1}^{(2)}-2 q_{2}^{(1)} \\
& =-2^{2} q_{2}^{(1)}-\left(1-2^{-1}\right) \sum_{\gamma^{\prime}=2}^{3} 2^{\gamma^{\prime}} q_{\gamma^{\prime}}^{(1)} \text {, } \\
& \lambda_{2,2}=\varepsilon^{(3)}+q_{1}^{(3)}-2 q_{2}^{(2)}=\varepsilon^{(4)}+q_{1}^{(4)}-2 q_{2}^{(2)} \\
& =-2^{2} q_{2}^{(2)}-\left(1-2^{-1}\right) \sum_{\gamma^{\prime}=2}^{3} 2^{\gamma^{\prime}} q_{\gamma^{\prime}}^{(2)}, \\
& \lambda_{3,1}=\varepsilon^{(1)}+q_{1}^{(1)}+2 q_{2}^{(1)}-4 q_{3}^{(1)} \\
& =\varepsilon^{(2)}+q_{1}^{(2)}+2 q_{2}^{(2)}-4 q_{3}^{(1)} \\
& =\varepsilon^{(3)}+q_{1}^{(3)}+2 q_{2}^{(2)}-4 q_{3}^{(1)} \\
& =\varepsilon^{(4)}+q_{1}^{(4)}+2 q_{2}^{(2)}-4 q_{3}^{(1)}=-2^{3} q_{3}^{(1)} \text {. }
\end{aligned}
$$

Here, $q_{\gamma}^{(n)}$ are nondiagonal elements of the PKM, while diagonal elements are $\varepsilon^{(m)}=-\left(1-2^{-1}\right) \sum_{\gamma=1}^{\Gamma} 2^{\gamma} q_{\gamma}^{(m)}$ by the definition of the kinetic matrix. Finally, eigenvector $\mathbf{e}\left(\gamma, n_{\gamma}\right)$ corresponds to eigenvalue $\lambda_{\gamma, n_{\gamma}}$.

Eigenvector $\mathbf{e}_{0}$ corresponds to eigenvalue $\lambda_{0}=0$. Comparing expressions (A.1) and (2), we can easily see that the expression

$$
\lambda_{\gamma, n}=-p^{\gamma} q_{\gamma}^{(n)}-\left(1-p^{-1}\right) \sum_{\gamma^{\prime}=\gamma+1}^{\Gamma} p^{\gamma^{\prime}} q_{\gamma^{\prime}}^{\left(n^{\prime}\right)}
$$

is a generalization of expressions (A.4) for arbitrary $p$ and $\Gamma$.

Let us now consider the structure of eigenvectors of a $9 \times 93$-adic PKM $(\Gamma=2)$. Level $\gamma=2$ is juxtaposed to a pair of complex-conjugate vectors:

$$
\mathbf{e}_{3}(2,1,1)=\left(\begin{array}{c}
1 \\
1 \\
1 \\
-\frac{1}{2}+i \frac{\sqrt{3}}{2} \\
-\frac{1}{2}+i \frac{\sqrt{3}}{2} \\
-\frac{1}{2}+i \frac{\sqrt{3}}{2} \\
-\frac{1}{2}-i \frac{\sqrt{3}}{2} \\
-\frac{1}{2}-i \frac{\sqrt{3}}{2} \\
-\frac{1}{2}-i \frac{\sqrt{3}}{2}
\end{array}\right) \text {, }
$$

$$
\mathbf{e}_{3}(2,1,2)=\left(\begin{array}{c}
1 \\
1 \\
1 \\
-\frac{1}{2}-i \frac{\sqrt{3}}{2} \\
-\frac{1}{2}-i \frac{\sqrt{3}}{2} \\
-\frac{1}{2}-i \frac{\sqrt{3}}{2} \\
-\frac{1}{2}+i \frac{\sqrt{3}}{2} \\
-\frac{1}{2}+i \frac{\sqrt{3}}{2} \\
-\frac{1}{2}+i \frac{\sqrt{3}}{2}
\end{array}\right) .
$$

Level $\gamma=1$ is juxtaposed to three pairs of complexconjugate vectors: 


$$
\begin{aligned}
& \mathbf{e}_{3}(1,1,1)=\left(\begin{array}{c}
1 \\
-\frac{1}{2}+i \frac{\sqrt{3}}{2} \\
-\frac{1}{2}-i \frac{\sqrt{3}}{2} \\
0 \\
0 \\
0 \\
0 \\
0 \\
0
\end{array}\right), \quad \mathbf{e}_{3}(1,1,2)=\left(\begin{array}{c}
1 \\
-\frac{1}{2}-i \frac{\sqrt{3}}{2} \\
-\frac{1}{2}+i \frac{\sqrt{3}}{2} \\
0 \\
0 \\
0 \\
0 \\
0 \\
0
\end{array}\right) \\
& \mathbf{e}_{3}(1,2,1)=\left(\begin{array}{c}
0 \\
0 \\
0 \\
1 \\
-\frac{1}{2}+i \frac{\sqrt{3}}{2} \\
-\frac{1}{2}-i \frac{\sqrt{3}}{2} \\
0 \\
0 \\
0
\end{array}\right), \quad \mathbf{e}_{3}(1,2,2)=\left(\begin{array}{c}
0 \\
0 \\
0 \\
1 \\
-\frac{1}{2}-i \frac{\sqrt{3}}{2} \\
-\frac{1}{2}+i \frac{\sqrt{3}}{2} \\
0 \\
0 \\
0
\end{array}\right) \\
& \mathbf{e}_{3}(1,3,1)=\left(\begin{array}{c}
0 \\
0 \\
0 \\
0 \\
0 \\
0 \\
1 \\
-\frac{1}{2}+i \frac{\sqrt{3}}{2} \\
-\frac{1}{2}-i \frac{\sqrt{3}}{2}
\end{array}\right), \quad \mathbf{e}_{3}(1,3,2)=\left(\begin{array}{c}
0 \\
0 \\
0 \\
0 \\
0 \\
0 \\
1 \\
-\frac{1}{2}-i \frac{\sqrt{3}}{2} \\
-\frac{1}{2}+i \frac{\sqrt{3}}{2}
\end{array}\right)
\end{aligned}
$$

Supplementing these eight eigenvectors with unit vector $\mathbf{e}_{0}$ forms the complete basis of symmetric $9 \times 9$ 3 -adic PKMs.

It should be noted in conclusion that translationinvariant PKMs correspond to the case where transition probabilities are functions of ultrametric distance alone (i.e., matrix elements of the PKMs are parametrized by the number of hierarchical level $\gamma$ ). As a result, eigenvalues $\lambda_{\gamma}$ are $(p-1) p^{\Gamma-\gamma}$-fold degenerate. The translation-noninvariant PKMs considered in Section 2 correspond to the case when the matrix elements of PKMs are parametrized only by $\left(\gamma, n_{\gamma}\right)$ pairs 
(i.e., they are independent of index $k$ ). In this case, each eigenvalue $\lambda_{\gamma, n_{\gamma}}$ is $(p-1)$-fold degenerate.

\section{REFERENCES}

1. K. Johansson, Commun. Math. Phys. 209, 437 (2000).

2. M. Praehofer and H. Spohn, Phys. Rev. Lett. 84, 4882 (2000).

3. M. L. Mehta, Random Matrices (Elsevier, Amsterdam, 2004).

4. M. Mezard, G. Parisi, and M. Virasoro, Spin Glass Theory and Beyond (World Sci., Singapore, 1987).

5. H. Frauenfelder, Nat. Struct. Biol. 2, 821 (1995).

6. V. S. Vladimirov, I. V. Volovich, and Y. I. Zelenov, P-Adic Analysis and Mathematical Physics (World Sci., Singapore, 1994).

7. S. Nechaev and R. Voituriez, J. Phys. A: Math. Gen. 34, 11069 (2001).

8. Y. V. Fyodorov and J.-P. Bouchaud, J. Phys. A: Math. Theor. 41, 324009 (2008).

9. A. Ogielsky and D. Stein, Phys. Rev. Lett. 55, 1634 (1985); C. P. Bachas and B. A. Huberman, Phys. Rev. Lett. 57, 1965 (1986).

10. G. Köhler and A. Blumen, J. Phys. A: Math. Gen. 20, 5627 (1987).

11. O. M. Becker and M. Karplus, J. Chem. Phys. 106, 1495 (1997)

12. V. A. Avetisov, A. Kh. Bikulov, and S. V. Kozyrev, J. Phys. A: Math. Gen. 32, 8785 (1999); G. Parisi and N. Sourlas, Eur. J. Phys. B 14, 535 (2000).

13. V. A. Avetisov, A. Kh. Bikulov, S. V. Kozyrev, and V. A. Osipov, J. Phys. A: Math. Gen. 35, 177 (2002).

14. V. A. Avetisov and A. Kh. Bikulov, Biophys. Rev. Lett. 3, 387 (2008).

15. V. A. Avetisov, A. Kh. Bikulov, and S. K. Nechaev, J. Phys. A: Math. Theor. 42, 075001 (2009).

16. A. Y. Grosberg, S. K. Nechaev, and E. I. Shakhnovich, J. Phys. (Paris) 49, 2095 (1988).

17. B. Mandelbrot, The Fractal Geometry of Nature (Freeman, New York, 1982).

18. V. A. Avetisov, A. Chertovich, S. K. Nechaev, and O. Vasilyev, arXiv:0811.4518; J. Stat. Mech. (2009) (in press).

19. R. Albert and A.-L. Barabási, Rev. Mod. Phys. 74, 47 (2002).

20. S. Kozyrev, V. Osipov, and V. Avetisov, J. Math. Phys. 46, 063302 (2005).

21. V. A. Avetisov, A. Kh. Bikulov, and V. A. Osipov, J. Phys. A: Math. Gen. 36, 4239 (2003).

22. B. Derrida and H. Spohn, J. Stat. Phys. 51, 817 (1988).

23. A. Kolmogorov, I. Petrovsky, and N. Piscounov, Moscow Univ. Bull. Math. 1, 1 (1937).

24. V. S. Dotsenko, Usp. Fiz. Nauk 163 (6), 1 (1993) [Phys. - Usp. 36 (6), 455 (1993)].

25. P. Erdös and A. Rényi, Publ. Math. Inst. Hung. Acad. Sci. A 5, 17 (1960).

26. G. J. Rodgers, K. Austin, B. Kahng, and D. Kim, J. Phys. A: Math. Gen. 38, 9431 (2005).

27. E. Ben-Naim and P. L. Krapivsky, Phys. Rev. E: Stat., Nonlinear, Soft Matter Phys. 71, 026129 (2005).
28. B. V. Chirikov and D. L. Shepelyansky, Physica D (Amsterdam) 13, 395 (1984).

29. J. D. Meiss and E. Ott, Physica D (Amsterdam) 20, 387 (1986).

30. A. V. Dobrynin, M. Rubinstein, and S. P. Obukhov, Macromolecules 29, 2974 (1996).

31. P. W. Fenimore, H. Frauenfelder, B. H. McMahon, and R. D. Young, Physica A (Amsterdam) 351, 1 (2005).

32. I. M. Lifshitz, Zh. Éksp. Teor. Fiz. 55 (6), 2408 (1968) [Sov. Phys. JETP 28 (6), 2408 (1968)].

33. I. M. Lifshitz, A. Y. Grosberg, and A. R. Khokhlov, Rev. Mod. Phys. 50, 683 (1978).

34. A. Y. Grosberg and A. R. Khokhlov, Statistical Physics of Macromolecules (American Institute of Physics, New York, 1994).

35. J. des Cloizeaux and G. Jannink, Polymers in Solution: Their Modelling and Structure (Oxford University Press, New York, 1990).

36. S. Nechaev and O. Vasilyev, J. Knot Theor. Ramifications 14, 243 (2005); S. Nechaev and O. Vasilyev, in Series on Knots and Everything: Physical and Numerical Models in Knot Theory, (World Sci., Singapore, 2005), Chapter 22, p. 421.

37. A. Grosberg, Y. Rabin, S. Havlin, and A. Neer, Europhys. Lett. 23, 373 (1993).

38. A. J. Einstein, H.-S. Wu, and J. Gil, Phys. Rev. Lett. 80, 397 (1998).

39. I. J. Farkas, I. Derényi, A.-L. Barabási, and T. Vicsek, Phys. Rev. E: Stat., Nonlinear, Soft Matter Phys. 64, 026704 (2001).

40. K.-I. Goh, B. Kahng, and D. Kim, Phys. Rev. E: Stat., Nonlinear, Soft Matter Phys. 64, 051903 (2001).

41. O. Bohigas, J. X. de Carvalho, and M. P. Pato, Phys. Rev. E: Stat., Nonlinear, Soft Matter Phys. 77, 011122 (2008).

42. A.-L. Barabási, E. Ravasz, and T. Vicsek, Physica A (Amsterdam) 299, 559 (2001).

43. A.-L. Barabási and R. Albert, Science (Washington) 286, 509 (1999); A.-L. Barabási, R. Albert, and H. Jeong, Physica A (Amsterdam) 272, 173 (1999); A.-L. Barabási, R. Albert, and H. Jeong, Physica A (Amsterdam) 281, 69 (2000).

44. F. Juhász, in Algebraic Methods in Graph Theory, Ed. by L. Lovasz and V. T. Sos (North-Holland, Amsterdam, 1981), p. 313; Z. Füredi and J. Komlös, Combinatorica 1, 233 (1981).

45. F. J. Dyson, J. Math. Phys. 3, 140 (1962); E. P. Wigner, SIAM Rev. 9, 1 (1967).

46. I. Farkas, I. Derányi, H. Jeong, Z. Néda, Z. N. Oltvai, E. Ravasz, A. Schubert, A.-L. Barabási, and T. Vicsek, Physica A (Amsterdam) 314, 25 (2002).

47. M. C. Gutzwiller and B. D. Mandelbrot, Phys. Rev. Lett. 60, 673 (1988).

48. P. Erdös, Am. J. Math. 61, 974 (1939).

49. B. Solomyak, Ann. Math. 142, 611 (1995).

50. N. Sidorov and A. Vershik, Monatsh. Math. 126, 215 (1998).

51. S. V. Kozyrev, Izv. Akad. Nauk, Ser. Mat. 66, 149 (2002).

Translated by N. Wadhwa 